\title{
Characteristics and genetic diversity of multi-drug resistant extended-spectrum beta-lactamase Escherichia coli isolated from bovine mastitis
}

\author{
Tariq Ali ${ }^{1, *}$, Sadeeq ur Rahman ${ }^{2, *}$, Limei Zhang ${ }^{1}$, Muhammad Shahid ${ }^{1}$, Dandan Han ${ }^{1}$, \\ Jian Gao ${ }^{1}$, Shiyao Zhang ${ }^{1}$, Pamela L. Ruegg ${ }^{3}$, Umer Saddique ${ }^{4}$ and Bo Han ${ }^{1}$ \\ ${ }^{1}$ Department of Clinical Veterinary Medicine, College of Veterinary Medicine, China Agricultural University, Beijing, P.R. China \\ ${ }^{2}$ College of Veterinary Sciences and Animal Husbandry, Abdul Wali Khan University, Garden Campus, Mardan, Pakistan \\ ${ }^{3}$ Department of Dairy Science, University of Wisconsin, Madison, WI, USA \\ ${ }^{4}$ Department of Animal Health, The University of Agriculture, Peshawar, Pakistan \\ *These authors have contributed equally and co-first authors
}

Correspondence to: Bo Han, email: hanbo@cau.edu.cn

Keywords: E. coli, ESBL, multilocus sequence typing, PCR-based replicon typing, split network analysis

Received: June 15,2017 Accepted: August 23, $2017 \quad$ Published: October 04, 2017

Copyright: Ali et al. This is an open-access article distributed under the terms of the Creative Commons Attribution License 3.0 (CC BY 3.0), which permits unrestricted use, distribution, and reproduction in any medium, provided the original author and source are credited.

\section{ABSTRACT}

A characterization of the drug resistance profiles, identification of PCR-based replicon typing, and multilocus sequence typing (MLST) and analysis of 46 ESBLproducing Escherichia coli from cows with mastitis are described. All multidrugresistant isolates of various phylogenetic groups $(A=31, B 1=3, B 2=2, D=10)$ were ESBL-producers of genotypes CTX-M-15 (29), CTX-M-55 (4), CTX-M-14 (4), CTX-M-3 (1), CTX-M-1 (1), TEM (22) and SHV (8) that were found on conjugative plasmids of diverse incompatibility groups (primarily IncF). Transconjugation experiments indicated successful $(\mathbf{1 0 0} \%)$ trans-conjugation, which was verified phenotypically and genotypically. A total of 28 sequence types (ST) were identified, with $10 \%$ of isolates being ST410, and 9 other ST that were assigned arbitrary numbers, reflecting the degree of diversity. Multilocus sequence analysis revealed two lineages, a dominant and a small lineage. Split-decomposition showed intraspecies recombination clearly contributed in genetic recombination generating genotypic diversity among the isolates, and a lack of interspecies recombination. This coherent analysis on genetic structure of multidrug-resistant pathogenic $E$. coli population isolated from mastiticmilk weaponized with resistance elements from a large, rapidly developing country will be a helpful contribution for epidemiology and surveillance of drug resistance patterns, and understanding their global diversity.

\section{INTRODUCTION}

Escherichia coli has been classified as a major pathogen causing bovine mastitis, inflammation of the mammary gland, due to its abundant and predominant isolation from infected tissues [1]. Other than mastitis, $E$. coli is also the most common pathogen responsible for several other serious diverse gastrointestinal or urinary tract infections, and even bacteremia in human beings, thereby causing millions of deaths every year around the globe. To eliminate this pathogen during infection, antimicrobial agents are administered for treatment, although in livestock these drugs may also be used in prophylactically manner, to prevent bacterial infections or promote livestock growth. To avoid mastitis-associated economic losses in dairy cows, potent drugs such as extended-spectrum cephalosporins are 
often preferred for treatment, this trend may be associated with emergence of bacterial resistance as indicated by the increasing prevalence of resistant extended-spectrum beta-lactamase (ESBL)-producing Enterobacteriaceae, especially E. coli, isolated from dairy and food-producing animals in China [2-4]. Notably, the emergence of novel antibacterial resistance mechanisms [5] and prevalence of multidrug resistant $E$. coli isolated from food animals have steadily increased over the last decades in China [6] and around the globe [7]. Although persistent and blind use of antimicrobials is likely the most important factor providing selective pressure for emergence of traits associated with resistance, understanding mechanisms of dissemination and genetic tools that help bacteria in spreading such elements are vital to control and elimination of antibiotic resistance.

Genes encoding ESBL have been classified into three major categories: $b l a_{\mathrm{CTX}-\mathrm{M}}, b l a_{\mathrm{SHV}}$ and $b l a_{\mathrm{TEM}}$. The $b l a_{\text {СТХ-М }}$ type has been further distributed into five main sub-groups ( $b l a_{\text {СTХ-M-1 }}, b l a_{\text {СТХ-M-2}}, b l a_{\text {СТХ-М-8 } 8}, b l a_{\text {СТХ-М- } 9}$, $b l a_{\mathrm{CTX}-\mathrm{M}-25}$ ) and different variants (approximately more than 150 CTX-M variants) have been documented thus far (http://www.lahey.org/studies). Previous reports suggest that the $b l a_{\text {СТХ-м }}$ type, predominantly $b l a_{\text {СтХ-М-15 }}$, was the most prevalent ESBL type around the world and especially in Asia [8]. Notably, E. coli producing bla ${ }_{\text {СтХ-М-15 }}$ ESBL is an important cause of health care oriented- as well as community based-infections in humans [9], and has also been increasingly reported recently from food producing animals [2]. It has been suggested that acquiring plasmids harbouring $b l a_{\text {СтХ-M }}$ and other ESBL encoding genes is the predominant mechanism associated with the increase of ESBL-producing E. coli. Importantly, ESBL genes on plasmids have been more frequently reported linked with certain plasmid replicon typing, including IncF, IncI, IncN, IncHI2, IncK and IncL/M groups [10]. The IncF group (FIA, FIB and FII) including IncK and IncI1 largely contribute to the dissemination of ESBL genes, primarily of $b l a_{\text {CTX-M -15 }}$, while the $b l a_{\text {СTХ-M -3 }}$ gene is carried by plasmids of IncL/M and IncI1, and IncHI2 plasmids harbour bla ${ }_{\text {СTX-M -9 }}[10]$.

For the purpose of precise identification and typing of E. coli, numerous molecular typing approaches have been developed including pulsed-field gel electrophoresis, restriction fragment length analysis, amplified fragment length fingerprinting, whole cell protein analysis, matrix-assisted laser desorption ionization, time of flight mass spectrometry, and multilocus sequence typing (MLST) [11-13]. Although all these methods can identify E. coli, MLST gives high resolution at specie level as compared to other molecular methods, and is accepted worldwide and widely used for typing of E. coli [14]. The MLST method for $E$. coli formulated in 2006 and updated in 2007 is used worldwide for typing and assessing the population structure of E. coli [15]. Furthermore, phylogenetic algorithm and procedures implemented in the multilocus sequence analysis (MLSA) based on the nucleotide sequences of allelic locus used in the MLST are being reported widely used for identification and inferring phylogenetic relationships between isolates. Moreover, MLST has been proven a powerful tool for insights into the population structure and recombination analysis of many bacterial pathogens including E. coli $[16,17]$. Nevertheless, MLST studies on drug resistant E. coli, especially ESBL-producing, isolated from food animals in general and particularly from mastitic dairy cows are lacking. We recently reported on the high prevalence of multi-drug resistant ESBL-producing $E$. coli from milk samples of dairy cows suffering from bovine mastitis [4]. In this study, we extended our analysis on the drug resistance profile including minimum inhibitory concentrations (MIC), plasmid replicon typing, and further provided insight based on the MLST of previously reported and newly isolated $E$. coli isolates. For the first time in China, E. coli isolates from bovine mastitic milk were identified as the species of $E$. coli by using MLSA, extrapolate about the recombination features and genetic diversity that reveals detailed insights into the prevailing drug resistant worldwide disseminated pathogen.

\section{RESULTS}

\section{Prevalence and genotypes of ESBL-producing $E$. coli}

As a part of our project, during 2015-2016 we screened 1440 milk samples from cows suffering from mastitis belonged to 69 different dairy herds in various provinces of China (Supplementary Table 1). Of these mastitic milk samples, a total of $181 \mathrm{E}$. coli isolates were recovered. Among these isolates, 46 were confirmed as extended-spectrum beta-lactamase (ESBL)-producers. Genotyping of these ESBL-producing E. coli by specific PCR assay showed that CTX-M producing genotypes were predominant $(n=39)$, followed by TEM $(n=$ $22)$ and SHV $(n=8)$. Further sequencing of CTX-M genotypes revealed that CTX-M-15 was the most dominant allele, which was harbored by 29 isolates, followed by CTX-M-55 $(n=4)$, CTX-M-14 $(n=4)$, CTX-M-3 $(n=2)$ and CTX-M-1 $(n=1)$. All TEM and SHV alleles were found identical to TEM-1 and SHV1 except one of the isolate was carrying singlet SHV12 obtained from a dairy cow in Heilongjiang province (Table 1). Genotypes TEM and SHV were found in combination with CTX-M-15 in the majority of isolates.

\section{Phylogenetic grouping}

Using triplex PCR assay, the 46 ESBL-producing $E$. coli were distributed to the phylogenetic groups A ( $n$ $=31), \mathrm{B} 1(n=3), \mathrm{B} 2(n=2)$ and $\mathrm{D}(n=10)$ as shown in Table 1. 
Table 1: Molecular characteristics and MLST typing of extended-spectrum beta-lactamase-producing $E$. coli isolated from bovine mastitis

\begin{tabular}{|c|c|c|c|c|c|c|c|c|}
\hline $\begin{array}{l}\text { Clinical } \\
\text { Isolates }\end{array}$ & Location & $\begin{array}{l}\text { ESBL } \\
\text { types }\end{array}$ & $\begin{array}{l}\text { Phylo- } \\
\text { groups }\end{array}$ & $\begin{array}{l}\text { Plasmid } \\
\text { replicon types }\end{array}$ & ST & STC & $\begin{array}{c}\text { Strain } \\
\text { deposited } \\
\text { in MLST } \\
\text { data base }\end{array}$ & $\begin{array}{c}\text { Resistance } \\
\text { profiles }\end{array}$ \\
\hline $1-\mathrm{G}$ & Guangdong & CTX-M-15 & A & $\begin{array}{c}\text { IncFrep, } \\
\text { IncHI2, IncP, } \\
\text { IncY }\end{array}$ & ST744 & non & HTS01G & $\begin{array}{c}\text { A, AC, CX, } \\
\text { CTX, CAZ,FEP, } \\
\text { AZT, N, CIP, C, } \\
\text { ST, TE }\end{array}$ \\
\hline $2-\mathrm{G}$ & Guangdong & $\begin{array}{c}\text { CTX-M-15, } \\
\text { TEM-1 }\end{array}$ & A & $\begin{array}{c}\text { IncHI2, IncK/B, } \\
\text { IncY }\end{array}$ & ST761 & non & - & $\begin{array}{c}\text { A, AC, CX, } \\
\text { CTX, CAZ,FEP, } \\
\text { AZT, N, C, ST, } \\
\text { TE }\end{array}$ \\
\hline 3-Im & $\begin{array}{c}\text { Inner } \\
\text { Mongolia }\end{array}$ & $\begin{array}{l}\text { TEM-1, } \\
\text { SHV-1 }\end{array}$ & $\mathrm{D}$ & IncHI1, IncK/B & ST156 & STC156 & - & $\begin{array}{c}\text { A, AC, CTX, N, } \\
\text { CIP, C, ST, TE }\end{array}$ \\
\hline 4-Im & $\begin{array}{c}\text { Inner } \\
\text { Mongolia }\end{array}$ & CTX-M-15 & A & IncFIA, IncFrep & ST906 & non & - & $\begin{array}{c}\text { A, AC, CX, } \\
\text { CTX, CAZ, } \\
\text { AZT, N, CIP, C, } \\
\text { ST, TE, G }\end{array}$ \\
\hline 5-Im & $\begin{array}{l}\text { Inner } \\
\text { Mongolia }\end{array}$ & $\begin{array}{c}\text { CTX-M-15, } \\
\text { TEM-1, } \\
\text { SHV-1 }\end{array}$ & $\mathrm{D}$ & $\begin{array}{c}\text { IncFIA, IncFIB, } \\
\text { IncK/B }\end{array}$ & ST2008 & non & HTS05IM & $\begin{array}{c}\text { A, AC, CX, } \\
\text { FOX, CTX, } \\
\text { CAZ,FEP, AZT, } \\
\text { N, CIP, ST, TE, } \\
\text { G }\end{array}$ \\
\hline 12-Im & $\begin{array}{l}\text { Inner } \\
\text { Mongolia }\end{array}$ & $\begin{array}{c}\text { CTX-M-15, } \\
\text { TEM-1 }\end{array}$ & B1 & $\begin{array}{l}\text { IncFIB, IncFIIs, } \\
\text { IncFrep, IncI1, } \\
\text { IncK/B }\end{array}$ & ST2008 & non & HTS12IM & $\begin{array}{c}\text { A, AC, CX, } \\
\text { FOX, CTX, } \\
\text { CAZ,FEP, AZT, } \\
\text { N, CIP, ST, TE, } \\
\text { G }\end{array}$ \\
\hline 14-Im & $\begin{array}{c}\text { Inner } \\
\text { Mongolia }\end{array}$ & $\begin{array}{c}\text { CTX-M-15, } \\
\text { SHV-1 }\end{array}$ & A & IncFrep,IncK/B & ST1121 & non & & $\begin{array}{c}\text { A, AC, CX, } \\
\text { CTX, CAZ, } \\
\text { AZT, N,C, CIP, } \\
\text { ST, TE, G }\end{array}$ \\
\hline 17-Im & $\begin{array}{c}\text { Inner } \\
\text { Mongolia }\end{array}$ & CTX-M-15 & $\mathrm{A}$ & $\begin{array}{l}\text { IncFIA, IncFIB, } \\
\text { IncHI2, IncN }\end{array}$ & ST361 & non & HTS17IM & $\begin{array}{c}\text { A, AC, CX, } \\
\text { CTX, CAZ,FEP, } \\
\text { AZT, N, CIP, ST, } \\
\text { TE, G }\end{array}$ \\
\hline 18-Im & $\begin{array}{l}\text { Inner } \\
\text { Mongolia }\end{array}$ & CTX-M-15 & A & $\begin{array}{l}\text { IncFIA, IncFIB, } \\
\text { IncFrep,IncK/B }\end{array}$ & ST361 & non & - & $\begin{array}{c}\text { A, AC, CX, } \\
\text { CTX, CAZ, } \\
\text { AZT, N, CIP, ST, } \\
\text { TE, G }\end{array}$ \\
\hline 19-Im & $\begin{array}{c}\text { Inner } \\
\text { Mongolia }\end{array}$ & CTX-M-15 & A & $\begin{array}{l}\text { IncFIA, IncFIB, } \\
\text { IncHI2, IncK/B }\end{array}$ & ST1121 & non & HTS19IM & $\begin{array}{c}\text { A, AC, CX, } \\
\text { CTX, CAZ,FEP, } \\
\text { AZT, N, CIP, ST, } \\
\text { TE, G }\end{array}$ \\
\hline 20-Im & $\begin{array}{l}\text { Inner } \\
\text { Mongolia }\end{array}$ & CTX-M-15 & $\mathrm{D}$ & IncFIA & ST468 & non & HTS20IM & \\
\hline 21-Im & $\begin{array}{c}\text { Inner } \\
\text { Mongolia }\end{array}$ & $\begin{array}{c}\text { CTX-M-15, } \\
\text { SHV-1 }\end{array}$ & $\mathrm{D}$ & IncFrep & ST3476 & non & HTS21IM & CTX, CIP, TE \\
\hline
\end{tabular}




\begin{tabular}{|c|c|c|c|c|c|c|c|c|}
\hline $\begin{array}{l}\text { Clinical } \\
\text { Isolates }\end{array}$ & Location & $\begin{array}{l}\text { ESBL } \\
\text { types }\end{array}$ & $\begin{array}{l}\text { Phylo- } \\
\text { groups }\end{array}$ & $\begin{array}{l}\text { Plasmid } \\
\text { replicon types }\end{array}$ & ST & STC & $\begin{array}{l}\text { Strain } \\
\text { deposited } \\
\text { in MLST } \\
\text { data base }\end{array}$ & $\begin{array}{c}\text { Resistance } \\
\text { profiles }\end{array}$ \\
\hline 22-Im & $\begin{array}{c}\text { Inner } \\
\text { Mongolia }\end{array}$ & $\begin{array}{l}\text { CTX-M-15, } \\
\text { SHV-1 }\end{array}$ & $\mathrm{D}$ & $\begin{array}{c}\text { IncFIB,IncFrep, } \\
\text { IncK/B }\end{array}$ & ST410 & STC23 & HTS22IM & $\begin{array}{c}\text { A, AC, CX, } \\
\text { FOX, CTX, } \\
\text { CAZ,FEP, AZT, } \\
\text { N, CIP, ST, TE, } \\
\text { G }\end{array}$ \\
\hline 23-Im & $\begin{array}{c}\text { Inner } \\
\text { Mongolia }\end{array}$ & $\begin{array}{l}\text { CTX-M-15, } \\
\text { SHV-1 }\end{array}$ & A & $\begin{array}{c}\text { IncFIA, IncFIB, } \\
\text { IncFrep, IncN, } \\
\text { IncK/B }\end{array}$ & ST1221 & non & HTS23IM & $\begin{array}{c}\text { A, AC, CX, } \\
\text { CTX, CAZ, } \\
\text { AZT, N,C, CIP, } \\
\text { ST, TE, G }\end{array}$ \\
\hline 25-Im & $\begin{array}{c}\text { Inner } \\
\text { Mongolia }\end{array}$ & $\begin{array}{l}\text { CTX-M-15, } \\
\text { TEM-1, } \\
\text { SHV-1 }\end{array}$ & A & $\begin{array}{l}\text { IncFIA, IncFIB, } \\
\text { IncFrep,IncK/B }\end{array}$ & ST361 & non & HTS25IM & $\begin{array}{c}\text { A, AC, CX, } \\
\text { FOX, CTX,FEP, } \\
\text { AZT, N, CIP, ST, } \\
\text { TE, G }\end{array}$ \\
\hline 28-Im & $\begin{array}{c}\text { Inner } \\
\text { Mongolia }\end{array}$ & $\begin{array}{l}\text { CTX-M-15, } \\
\text { TEM-1 }\end{array}$ & A & $\begin{array}{c}\text { IncFIB,IncFrep, } \\
\text { IncP }\end{array}$ & ST3044 & non & - & $\begin{array}{c}\text { A, AC, CX, } \\
\text { FOX, CTX, } \\
\text { CAZ, AZT, N, } \\
\text { CIP, ST, TE, G }\end{array}$ \\
\hline 32-Im & $\begin{array}{c}\text { Inner } \\
\text { Mongolia }\end{array}$ & CTX-M-15 & A & $\begin{array}{l}\text { IncFIB,IncFrep, } \\
\text { IncHI2, IncN }\end{array}$ & ST2008 & non & - & $\begin{array}{c}\text { A, AC, CX, } \\
\text { CTX, CAZ,FEP, } \\
\text { C }\end{array}$ \\
\hline $1-\operatorname{Im} 2$ & $\begin{array}{c}\text { Inner } \\
\text { Mongolia }\end{array}$ & CTX-M-14 & A & IncI1, IncY & ST2521 & non & HTS01IM2 & $\begin{array}{c}\mathrm{A}, \mathrm{CX}, \mathrm{CTX}, \\
\mathrm{AZT},\end{array}$ \\
\hline $2-\operatorname{Im} 2$ & $\begin{array}{c}\text { Inner } \\
\text { Mongolia }\end{array}$ & TEM-1 & A & IncHI1, IncFIB & ST4085 & non & HTS02IM2 & A, CX, CTX, G \\
\hline $3-\operatorname{Im} 2$ & $\begin{array}{c}\text { Inner } \\
\text { Mongolia }\end{array}$ & TEM-1 & A & $\begin{array}{c}\text { IncFIA, IncFIB, } \\
\text { IncFrep }\end{array}$ & ST392 & non & HTS03IM2 & CX, CTX, AZT \\
\hline 4-Im2 & $\begin{array}{c}\text { Inner } \\
\text { Mongolia }\end{array}$ & CTX-M-15 & A & $\begin{array}{c}\text { IncFIB,IncFrep, } \\
\text { IncHI } 2\end{array}$ & ST1080 & non & HTS04IM2 & $\begin{array}{l}\text { A, CX, CTX, } \\
\text { AZT, C, ST, G }\end{array}$ \\
\hline $5-\operatorname{Im} 2$ & $\begin{array}{c}\text { Inner } \\
\text { Mongolia }\end{array}$ & CTX-M-15 & $\mathrm{A}$ & IncFIB, IncFrep & ST2035 & non & HTS05IM2 & $\begin{array}{c}\text { A, CX, CTX, } \\
\text { AZT, N }\end{array}$ \\
\hline 7-Im2 & $\begin{array}{c}\text { Inner } \\
\text { Mongolia }\end{array}$ & $\begin{array}{l}\text { CTX-M-55, } \\
\text { TEM-1 }\end{array}$ & B1 & $\begin{array}{l}\text { IncFIB,IncFrep, } \\
\text { IncN }\end{array}$ & ST410 & STC23 & HTS07IM2 & $\begin{array}{c}\text { A, AC, CX, } \\
\text { CTX, CAZ,FEP, } \\
\text { AZT, N, C, CIP, } \\
\text { ST, TE, G }\end{array}$ \\
\hline 8-Im2 & $\begin{array}{c}\text { Inner } \\
\text { Mongolia }\end{array}$ & CTX-M-14 & A & IncFIB, IncFrep & ST410 & STC23 & - & $\begin{array}{c}\text { A, CX, CTX, } \\
\text { CAZ,FEP, AZT, } \\
\text { N, C, CIP, ST, } \\
\text { TE, G }\end{array}$ \\
\hline 9-Im2 & $\begin{array}{c}\text { Inner } \\
\text { Mongolia }\end{array}$ & $\begin{array}{l}\text { CTX-M-55, } \\
\text { TEM-1 }\end{array}$ & A & $\begin{array}{l}\text { IncFIB,IncFrep, } \\
\text { IncN }\end{array}$ & ST410 & STC23 & - & $\begin{array}{c}\text { A, CX, CTX, } \\
\text { CAZ,FEP, AZT, } \\
\text { N, C, ST, TE, G }\end{array}$ \\
\hline $10-\operatorname{Im} 2$ & $\begin{array}{c}\text { Inner } \\
\text { Mongolia }\end{array}$ & $\begin{array}{l}\text { CTX-M-15, } \\
\text { TEM-1 }\end{array}$ & A & $\begin{array}{l}\text { IncFIA, IncFIB, } \\
\text { IncFrep, IncH2 }\end{array}$ & ST4085 & non & - & $\begin{array}{c}\text { A, CX, CTX, } \\
\text { CAZ, AZT, N, C, } \\
\text { CIP, ST, TE, G }\end{array}$ \\
\hline & & & & & & & & (Continued) \\
\hline
\end{tabular}




\begin{tabular}{|c|c|c|c|c|c|c|c|c|}
\hline $\begin{array}{l}\text { Clinical } \\
\text { Isolates }\end{array}$ & Location & $\begin{array}{l}\text { ESBL } \\
\text { types }\end{array}$ & $\begin{array}{l}\text { Phylo- } \\
\text { groups }\end{array}$ & $\begin{array}{l}\text { Plasmid } \\
\text { replicon types }\end{array}$ & ST & STC & $\begin{array}{c}\text { Strain } \\
\text { deposited } \\
\text { in MLST } \\
\text { data base }\end{array}$ & $\begin{array}{c}\text { Resistance } \\
\text { profiles }\end{array}$ \\
\hline 11-Im2 & $\begin{array}{c}\text { Inner } \\
\text { Mongolia }\end{array}$ & TEM-1 & B1 & $\begin{array}{l}\text { IncFIB,IncFrep, } \\
\text { IncH2 }\end{array}$ & ST4085 & non & HTS11IM2 & $\begin{array}{c}\text { A, AC, CX, } \\
\text { CTX, AZT, N, C } \\
\text { ST, TE, G }\end{array}$ \\
\hline 12-Im2 & $\begin{array}{c}\text { Inner } \\
\text { Mongolia }\end{array}$ & CTX-M-55 & A & $\begin{array}{c}\text { IncFIB, } \\
\text { IncFrep, IncH2 }\end{array}$ & ST215 & STC10 & - & $\begin{array}{c}\text { A, CX, } \\
\text { CTX,FEP, AZT, } \\
\text { N, C, CIP, TE }\end{array}$ \\
\hline $8-\operatorname{Im} 3$ & $\begin{array}{c}\text { Inner } \\
\text { Mongolia }\end{array}$ & CTX-M-55 & $\mathrm{D}$ & $\begin{array}{l}\text { IncFIB, IncFIC, } \\
\text { IncFrep }\end{array}$ & ST5746 & non & HTS08IM3 & $\begin{array}{c}\text { A, AC, CX, } \\
\text { CTX, AZT, N, C } \\
\text { ST, TE, G }\end{array}$ \\
\hline $1-\operatorname{Im} 5$ & $\begin{array}{c}\text { Inner } \\
\text { Mongolia }\end{array}$ & $\begin{array}{l}\text { CTX-M-15, } \\
\text { TEM-1 }\end{array}$ & A & IncFrep & ST744 & non & HTS01IM5 & $\begin{array}{c}\text { A, AC, CX, } \\
\text { CTX, CAZ,FEP, } \\
\text { AZT, N, C, CIP, } \\
\text { ST, TE, G }\end{array}$ \\
\hline $2-\operatorname{Im} 5$ & $\begin{array}{c}\text { Inner } \\
\text { Mongolia }\end{array}$ & TEM-1 & A & $\begin{array}{c}\text { IncFIB, } \\
\text { IncFrep, IncH2 }\end{array}$ & ST4085 & non & HTS02IM5 & $\begin{array}{c}\text { A, AC, CX, } \\
\text { FOX, CTX, } \\
\text { CAZ,FEP, AZT, } \\
\text { N, C, CIP, ST, } \\
\text { TE, G }\end{array}$ \\
\hline $3-\operatorname{Im} 5$ & $\begin{array}{c}\text { Inner } \\
\text { Mongolia }\end{array}$ & $\begin{array}{l}\text { CTX-M-14, } \\
\text { TEM-1 }\end{array}$ & A & $\begin{array}{l}\text { IncFIA, IncFIB, } \\
\text { IncFrep, IncP }\end{array}$ & ST58 & STC155 & HTS03IM5 & $\begin{array}{l}\text { A, AC, CX, } \\
\text { FOX, CTX, N, } \\
\text { ST, TE, G }\end{array}$ \\
\hline 1-Im6 & $\begin{array}{c}\text { Inner } \\
\text { Mongolia }\end{array}$ & CTX-M-15 & B2 & IncFIB, IncFrep & ST117 & non & HTS01IM6 & $\begin{array}{c}\text { A, AC, CX, } \\
\text { CTX, CAZ,FEP, } \\
\text { AZT, N, C, ST, } \\
\text { TE, G }\end{array}$ \\
\hline $2-\operatorname{Im} 6$ & $\begin{array}{c}\text { Inner } \\
\text { Mongolia }\end{array}$ & CTX-M-15 & B2 & IncFIB, IncFrep & ST117 & non & - & $\begin{array}{c}\text { A, AC, CX, } \\
\text { CTX, CAZ,FEP, } \\
\text { AZT, N, C, CIP, } \\
\text { ST, TE, G }\end{array}$ \\
\hline $4-\mathrm{N}$ & Jiangsu & $\begin{array}{l}\text { CTX-M-15, } \\
\text { TEM-1 }\end{array}$ & $\mathrm{D}$ & IncI1, IncY & ST88 & STC23 & HTS04N & $\begin{array}{c}\text { A, CX, CTX, } \\
\text { CAZ,FEP, AZT, } \\
\text { N, C, ST, TE, G }\end{array}$ \\
\hline $5-\mathrm{N}$ & Jiangsu & $\begin{array}{l}\text { CTX-M-15, } \\
\text { TEM-1 }\end{array}$ & D & IncI1, IncY & ST88 & STC23 & - & $\begin{array}{c}\text { A, CX, CTX, } \\
\text { CAZ,FEP, AZT, } \\
\text { N, C, ST, TE, G }\end{array}$ \\
\hline $4-\mathrm{Hb}_{2}$ & Hebei & TEM-1 & $\mathrm{D}$ & $\begin{array}{l}\text { IncFIA, IncY, } \\
\text { IncL/M }\end{array}$ & ST69 & STC69 & - & $\begin{array}{c}\text { A, AC, CX, } \\
\text { CTX, CAZ,FEP, } \\
\text { AZT, N, C, ST, } \\
\text { TE, G }\end{array}$ \\
\hline $1-\mathrm{Hb}_{3}$ & Hebei & CTX-M-15 & A & IncFIB, IncFrep & ST58 & STC155 & HTS01HB & $\begin{array}{c}\text { A, AC, CX, } \\
\text { CTX, CAZ,FEP, } \\
\text { AZT, N, C, CIP, } \\
\text { ST, TE, G }\end{array}$ \\
\hline
\end{tabular}

(Continued) 


\begin{tabular}{|c|c|c|c|c|c|c|c|c|}
\hline $\begin{array}{l}\text { Clinical } \\
\text { Isolates }\end{array}$ & Location & $\begin{array}{l}\text { ESBL } \\
\text { types }\end{array}$ & $\begin{array}{l}\text { Phylo- } \\
\text { groups }\end{array}$ & $\begin{array}{c}\text { Plasmid } \\
\text { replicon types }\end{array}$ & ST & STC & $\begin{array}{c}\text { Strain } \\
\text { deposited } \\
\text { in MLST } \\
\text { data base }\end{array}$ & $\begin{array}{c}\text { Resistance } \\
\text { profiles }\end{array}$ \\
\hline $1-\mathrm{L}$ & Liaoning & $\begin{array}{c}\text { CTX-M-15, } \\
\text { TEM-1 }\end{array}$ & A & IncFIB, IncN & ST117 & non & HTS01L & $\begin{array}{c}\text { A, AC, CX, } \\
\text { CTX, CAZ,FEP, } \\
\text { AZT, N, C, ST, } \\
\text { TE, G }\end{array}$ \\
\hline 8-L2 & Liaoning & CTX-M-14 & A & IncFIB, IncHI2 & ST3951 & non & - & $\begin{array}{c}\text { A, AC, CX, } \\
\text { CTX, CAZ, } \\
\text { AZT, N, ST, TE }\end{array}$ \\
\hline N-1 & Ningxia & $\begin{array}{l}\text { CTX-M-3, } \\
\text { TEM-1 }\end{array}$ & A & IncFIA, IncI1 & NT & & & $\begin{array}{c}\text { A, AC, CX, } \\
\text { CTX, N, C, TE }\end{array}$ \\
\hline H-5 & Heilongjiang & SHV-12 & A & IncFIB, & NT & & & $\begin{array}{c}\mathrm{A}, \mathrm{CX}, \mathrm{FOX}, \\
\mathrm{CTX}\end{array}$ \\
\hline Hn-6 & Henan & CTX-M-15 & A & IncFrep, IncK/B & NT & & & $\begin{array}{c}\mathrm{A}, \mathrm{AC}, \mathrm{CX}, \\
\mathrm{FOX}, \mathrm{CTX}, \mathrm{C}, \\
\mathrm{TE}\end{array}$ \\
\hline 2-Hn1 & Henan & CTX-M-1 & A & $\begin{array}{l}\text { IncFIB, IncFIC, } \\
\text { IncK/B }\end{array}$ & ST58 & STC155 & - & $\begin{array}{c}\text { A, CX, CTX, } \\
\text { CAZ,FEP, AZT, } \\
\text { N, C, G }\end{array}$ \\
\hline 6-Hn1 & Henan & $\begin{array}{c}\text { CTX-M-15, } \\
\text { TEM-1 }\end{array}$ & A & IncFIB, IncK/B & ST6482 & non & - & $\begin{array}{c}\text { A, AC, CX, } \\
\text { CTX, CAZ,FEP, } \\
\text { AZT, N, ST, } \\
\text { TE, G }\end{array}$ \\
\hline 7-Hn1 & Henan & $\begin{array}{c}\text { CTX-M-15, } \\
\text { TEM-1 }\end{array}$ & A & $\begin{array}{l}\text { IncFIA, IncFIB, } \\
\text { IncFrep, IncK/B }\end{array}$ & ST540 & non & HTS07HN & $\begin{array}{c}\text { A, AC, CX, } \\
\text { FOX, CTX, } \\
\text { CAZ,FEP, AZT, } \\
\text { N, ST, TE, G }\end{array}$ \\
\hline
\end{tabular}

NT: nontypeable; A: ampicillin, AC: amoxicillin/clavulanic acid, CX: cephalexin, FOX: cefoxatin, CTX: cefotaxime, CAZ: ceftazidime, FEP: cefepime, AZT: aztreonam, N: nalidixic acid, ST: trimethoprim/sulphamethoxazole, TE: tetracycline, GM: gentamicin.

\section{Antimicrobial resistance in ESBL-producers}

Dairy cattle with mastitis are treated with broad spectrum antibiotics, so we screened these isolates for resistance against commonly used antibiotics. Interestingly, all the 46 ESBL-producing $E$. coli were multidrug resistant phenotypes (i.e., showing resistance to three or more classes of antimicrobial agents) by standard disc diffusion (Figure 1) and MIC (Table 2). On observing the antibiotic susceptibility patterns of ESBL-producers, all isolates were non-susceptible to cefotaxime, while $32(66.67 \%)$ and $28(58.33 \%)$ isolates were resistant to ceftazidime and cefepime, respectively. Resistance to other classes of antimicrobial agents was also greater (Figure 1). However, all isolates were susceptible to meropenem and $38(79.17 \%)$ isolates were susceptible to cefoxatin (MIC of various drugs tested are shown in Table
2), confirming the level of resistance determined initially by double disc synergy test. Isolates under study were resistant revealing highest values of MIC 50 and MIC 90 of common drugs in clinical practice such as ampicillin, cefotaxime, ceftriaxone, tetracycline, gentamycin etc. (Table 2).

\section{PCR-based plasmid replicon typing}

Identification of the plasmid replicon typing for epidemiological studies is quite important. The results of PCR-based replicon typing of all the ESBL-producing $E$. coli revealed that $\mathrm{IncF}$ was the major plasmid replicon type identified in 41 isolates, whereas IncL/M was detected in only one isolate. Figure 2 shows the results of plasmid replicon types of some of the ESBL-producing $E$. coli isolates from bovine mastitis. The plasmids belong 
to the incompatibility groups IncFIA $(n=14)$, IncFIB $(n=31)$, IncFIC $(n=2)$, IncFrep $(n=29)$, IncFIIs $(n=$ $1)$, IncH1 $(n=2), \operatorname{IncH} 2(n=11)$, IncI1 $(n=5)$, IncK/B $(n=12)$, IncN $(n=6), \operatorname{IncL} / \mathrm{M}(n=1)$ and IncY $(n=$ 8 ) as shown in the Table 1. Interestingly, in the majority of isolates multiple plasmids replicon types were found except in three ESBL-producing isolates, which carried a single plasmid replicon type.

\section{Resistance transfer experiment}

Presence of the drug resistance elements such as $b l a_{\text {СТХ-м }}$ on conjugative plasmids is expected to facilitate fast dissemination of these elements. Thus, isolates under study were further tested by mating/ conjugation experiments to verify the transferability and possible horizontal spread of ESBL genes and plasmids. For this purpose, we randomly tested twelve isolates. All resulting transconjugates displayed features of multidrug resistant phenotypes as of donor (Table 3 ). Further, the transconjugates also displayed greater MIC of cefotaxime and ceftazidime. ESBL encoding genes were PCR-amplified from the transconjugates suggesting presence of ESBL genes on conjugative plasmids. Plasmid replicon typing using PCR was also carried out on all transconjugates, which showed that plasmid incompatibility type incF was found in all transconjugates except the two (Trans-1-Im2 and Trans-4N) that carried incY (Table 3).

\section{Multilocus typing and population structure}

A total of 28 sequence types (ST), including 9 possibly novel ST, were identified among 43 typeable $E$. coli multidrug resistant isolates recovered from mastitic milk. Three of the E. coli isolates could not be assigned with a ST as we were not able to amplify at least one of the house keeping genes. Location from where these isolates were obtained, genes conferring resistance to target drugs, plasmid replicon, ST and antibiogram are mentioned in Table 1. Furthermore, ST identified along with allelic profile of 43 typeable $E$. coli isolates are given in Table 4.

The overall epidemiological information of the prevailing ST based on the MLST data of all isolates $(n=43)$ revealed a total of $28 \mathrm{ST}$, of which, $9 \mathrm{ST}$ were assigned with arbitrary types (10000-08) and 19 ST were matched with the MLST database of $E$. coli. Sequence types $410(10 \%), 361(7 \%), 4085$ (7\%), $117(7 \%), 58$ (7\%), 744 (5\%), $2008(5 \%)$ and $1121(5 \%)$ were the most prevalent, while ST 761, 5442, 3476, 2521, 392, 1080, $2035,215,5746,88$ and 69 were the least (2\%) prevalent. Interestingly, all those ST (10000-08) that could not be assigned as a result of at least a singular novel allele were all found among the least prevalent (2\%) ST. Details about the distribution and abundance of ST are described in Figure 3A. The most prevalent ST (ST410) originated from Inner Mongolia, and 6 of the 9 ST that were not assigned based on the allelic profile also originated

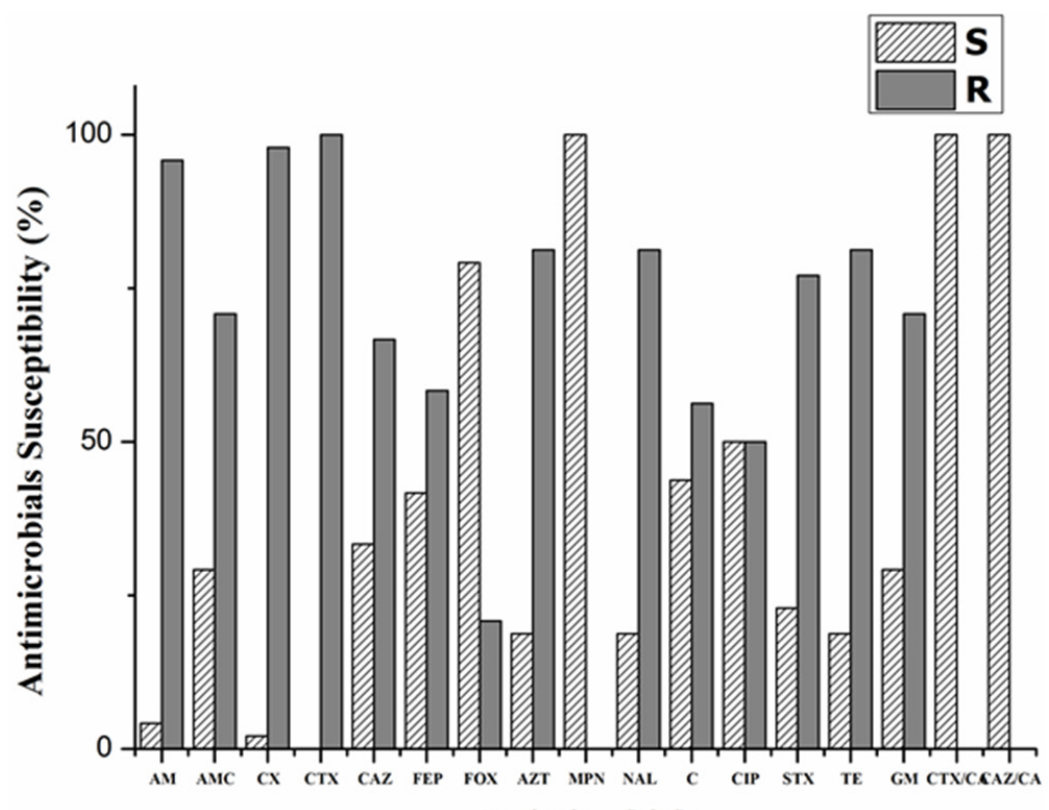

Antimicrobials

Figure 1: Antibiotic susceptibility profiles and PCR-based plasmid replicon typing of extended-spectrum betalactamase-producing $\boldsymbol{E}$. coli isolates from bovine mastitis. Results of antimicrobials susceptibility testing of ESBL-producing representative E. coli isolates are shown. The isolate(s) was tested for ESBL-production as described by CLSI. AM: ampicillin, AMC: amoxicillin/clavulanic acid, CX: cephalexin, cefaclor, CTX: cefotaxime, CAZ: ceftazidime, FEP: cefepime, FOX: cefoxatin, AZT: aztreonam, MPN: meropenem, NAL: nalidixic acid, C: chloramphenicol, CIP: ciprofloxacin, STX: trimethoprim/sulphamethoxazole, TE: tetracycline, GM: gentamicin, CTX/CA: cefotaxime/ clavulanic acid and CAZ/CA: ceftazidime/clavulanic acid. 
Table 2: Minimum inhibitory concatenations (MIC) of ESBL-producing $E$. coli $(n=46)$

\begin{tabular}{lcccccc}
\hline $\begin{array}{l}\text { Antimicrobial } \\
\text { agents }\end{array}$ & $\begin{array}{c}\text { Break-points } \\
(\mathbf{u g} / \mathbf{m l})\end{array}$ & $\begin{array}{c}\mathbf{M I C} \text { range } \\
(\mathbf{u g} / \mathbf{m l})\end{array}$ & $\mathbf{M I C}_{\mathbf{5 0}}(\mathbf{u g} / \mathbf{m l})$ & $\mathbf{M I C}_{\mathbf{9 0}}(\mathbf{u g} / \mathbf{m l})$ & $\begin{array}{c}\text { No. of } \\
\text { susceptible } \\
\text { isolates (\%) }\end{array}$ & $\begin{array}{c}\text { No. of resistant } \\
\text { isolates (\%) }\end{array}$ \\
\hline Ampicillin & $\geq 32$ & $0.5->256$ & $>256$ & $>256$ & $1(2.17)$ & $45(97.83)$ \\
Cefazolin & $\geq 8$ & $0.5->256$ & $>256$ & $>256$ & $2(4.35)$ & $44(95.65)$ \\
Cefoxatin & $\geq 4$ & $0.5->256$ & $>1$ & $>64$ & $31(67.39)$ & $15(32.619)$ \\
Cefotaxime & $\geq 4$ & $0.5->256$ & $>256$ & $>256$ & $0(00)$ & $46(100)$ \\
Ceftriaxone & $\geq 4$ & $0.5->256$ & $>256$ & $>256$ & $2(4.35)$ & $44(95.65)$ \\
Ciprofloxacin & $\geq 4$ & $0.5->256$ & $>2$ & $>256$ & $22(47.83)$ & $24(52.17)$ \\
Norfloxacin & $\geq 16$ & $0.5->256$ & $>32$ & $>256$ & $20(43.48)$ & $26(56.52)$ \\
Amikacin & $\geq 64$ & $0.5->256$ & $>64$ & $>128$ & $21(45.65)$ & $25(54.35)$ \\
Gentamicin & $\geq 16$ & $0.5->256$ & $>128$ & $>256$ & $10(21.74)$ & $36(78.26)$ \\
Kanamycin & $\geq 64$ & $0.5->256$ & $>128$ & $>256$ & $10(21.74)$ & $36(78.26)$ \\
Tetracycline & $\geq 16$ & $0.5->256$ & $>256$ & $>256$ & $6(13.04)$ & $40(86.96)$ \\
Chloramphenicol & $\geq 32$ & $0.5->256$ & $>128$ & $>256$ & $15(32.61)$ & $31(67.39)$ \\
Trimethoprim & $\geq 16$ & $0.5->256$ & $>256$ & $>256$ & $8(17.39)$ & $38(82.61)$ \\
\hline
\end{tabular}

${ }^{\mathrm{a} C L S I}, 2015$.

from Inner Mongolia. Furthermore, both of the isolates (ST10005 and ST10006) that could not be assigned to a ST originated from Jiangsu province.

All 28 ST found in the current report were grouped into 5 BURST group (BG) and 17 singletons types by eBURST analysis (Supplementary Table 3 ). The major BG contained 6 isolates and 3 ST $(88,410$ and 10005) with a predicted founder of ST88. A second BG consisted of 3 isolates and 2 ST (744 and 761) with no predicted founder. BG 3 was comprised of 3 isolates and 2 ST
(10007 and 540) with no predicted founder, BG4 was comprised of 3 isolates and 2 ST $(2008,10004)$ and BG 5 also comprised of 3 isolates and 2 ST (1121 and 10002), respectively (Supplementary Table 3 ). The geoBURST analysis with all $7256 \mathrm{ST}$ available in the PubMLST database (as of March 15, 2017) including ST under this report (Figure 3B) revealed that ST2035 was linked to ST10002, ST2035, ST898 (Founder), ST1121 (founder) and ST3519 (founder). Interestingly, ST88 (encircled in Figure 3B) that was isolated in this study was found

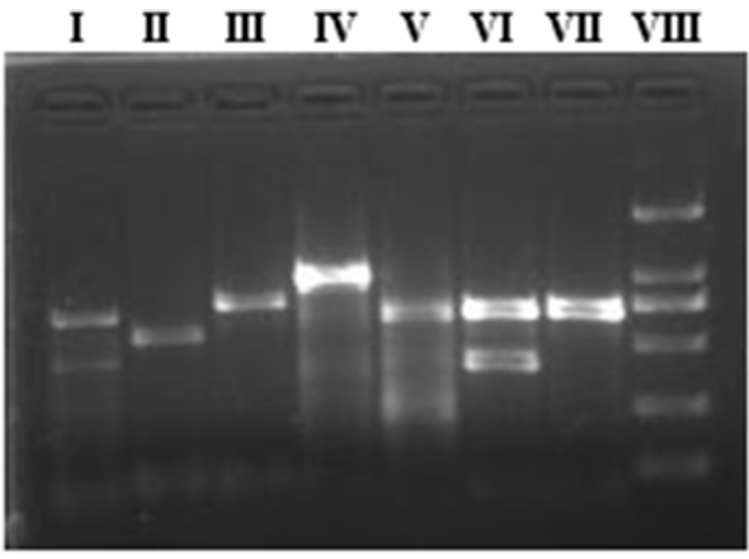

2000 bp

$1000 \mathrm{bp}$

$750 \mathrm{bp}$

500 bp

$250 \mathrm{bp}$

$100 \mathrm{bp}$

Figure 2: Detection of plasmid replicon types in ESBL-producing $\boldsymbol{E}$. coli using PCR assay. The amplified products were run on $2 \%$ agarose gel. Lane I= IncFIB and IncIncFIA, Lane II $=$ IncN, Lane III $=$ IncY, Lane IV $=$ Inc L/M, Lane V = IncHI2, Lane VI = IncFIB and IncHI1, Lane VII = IncFIB, and Lane VIII = molecular marker (2000 bp). 
Table 3: Characteristics of transconjugates using $E$. coli J53 strain of $(n=12)$

\begin{tabular}{|c|c|c|c|c|c|c|}
\hline Transconjugates & $\begin{array}{c}\text { ESBL } \\
\text { phenotype by } \\
\text { DDST }\end{array}$ & $\begin{array}{c}\text { ESBL } \\
\text { genotypes }\end{array}$ & $\begin{array}{l}\text { Plasmid } \\
\text { replicons }\end{array}$ & $\begin{array}{c}\text { CTX MIC } \\
(\mathrm{mg} / \mathrm{l})\end{array}$ & $\begin{array}{c}\text { CAZ MIC } \\
(\mathrm{mg} / \mathrm{l})\end{array}$ & $\begin{array}{l}\text { Resistance } \\
\text { phenotype }\end{array}$ \\
\hline Trans-1G & ESBL & $\begin{array}{c}\text { CTX-M-15, } \\
\text { TEM-1 }\end{array}$ & IncFrep & $>256$ & $>64$ & $\begin{array}{l}\text { A, AC, CX, } \\
\text { CTX, CAZ, } \\
\text { FEP, AZT, N, } \\
\text { CIP, TE }\end{array}$ \\
\hline Trans-5-Im & ESBL & CTX-M-15 & IncFIA, IncFIB & $>256$ & $>128$ & $\begin{array}{c}\text { A, AC, CX, } \\
\text { FOX, CTX, } \\
\text { CAZ, FEP, } \\
\text { AZT, ST }\end{array}$ \\
\hline Trans-12-Im & ESBL & CTX-M-15 & IncFIB & $>256$ & $>64$ & $\begin{array}{c}\text { A, CX, FOX, } \\
\text { CTX, CAZ, } \\
\text { FEP, AZT, CIP, } \\
\text { ST, GM }\end{array}$ \\
\hline Trans-17-Im & ESBL & CTX-M-15 & IncFIA, IncFIB & $>256$ & $>128$ & $\begin{array}{c}\text { A, CX, CTX, } \\
\text { CAZ,FEP, AZT, } \\
\text { CIP, TE, GM }\end{array}$ \\
\hline Trans-18-Im & ESBL & CTX-M-15 & IncFIB & $>256$ & $>32$ & $\begin{array}{c}\text { A, AC, CX, } \\
\text { CTX, CAZ, } \\
\text { AZT, N, CIP, } \\
\text { ST, TE, GM }\end{array}$ \\
\hline Trans-19-Im & ESBL & CTX-M-15 & IncFIA, IncFIB & $>256$ & $>128$ & $\begin{array}{c}\text { A, CX, CTX, } \\
\text { CAZ, FEP, } \\
\text { AZT, G }\end{array}$ \\
\hline Trans-25-Im & ESBL & $\begin{array}{c}\text { CTX-M-15, } \\
\text { TEM-1 }\end{array}$ & IncFIA, IncFIB & $>256$ & $>0.5$ & $\begin{array}{l}\text { A, CX, FOX, } \\
\text { CTX, FEP, } \\
\text { AZT, N, CIP, } \\
\text { ST, TE, GM }\end{array}$ \\
\hline Trans-28-Im & ESBL & CTX-M-15 & IncFrep & $>256$ & $>256$ & $\begin{array}{c}\text { A, AC, CX, } \\
\text { FOX, CTX, } \\
\text { CAZ, AZT, N, } \\
\text { CIP, ST, TE, } \\
\text { GM }\end{array}$ \\
\hline Trans-1-Im2 & ESBL & CTX-M-14 & IncY & $>16$ & $>1$ & $\begin{array}{c}\mathrm{A}, \mathrm{CX}, \mathrm{CTX}, \\
\mathrm{AZT}\end{array}$ \\
\hline Trans-4-N & ESBL & $\begin{array}{c}\text { CTX-M-15, } \\
\text { TEM-1 }\end{array}$ & IncY & $>256$ & $>64$ & $\begin{array}{c}\text { A, CX, CTX, } \\
\text { CAZ, FEP, } \\
\text { AZT, ST, TE, } \\
\text { GM }\end{array}$ \\
\hline Trans-6-Hn & ESBL & CTX-M-15 & IncFrep & $>64$ & $>1$ & $\begin{array}{l}\text { A, CX, FOX, } \\
\text { CTX, C, TE }\end{array}$ \\
\hline Trans-8-L2 & ESBL & CTX-M-14 & IncFIB & $>256$ & $>64$ & $\begin{array}{c}\text { A, CX, CTX, } \\
\text { CAZ, AZT, TE }\end{array}$ \\
\hline
\end{tabular}

A: ampicillin, AC: amoxicillin/clavulanic acid, CX: cephalexin, FOX: cefoxatin, CTX: cefotaxime, CAZ: ceftazidime, AZT: aztreonam, N: nalidixic acid, ST: trimethoprim/sulphamethoxazole, TE: tetracycline, GM: gentamicin. 
Table 4: $E$. coli isolates collection used in this study, their allele profiles and sequence type identified

\begin{tabular}{|c|c|c|c|c|c|c|c|c|c|}
\hline ID & ST & STC & $a d k$ & fumc & $\operatorname{gyr} B$ & icd & $m d h$ & purA & $\operatorname{rec} A$ \\
\hline $1-G$ & 744 & Non & 10 & 11 & 135 & 8 & 8 & 8 & 2 \\
\hline $2-\mathrm{G}$ & 761 & Non & 10 & 11 & 5 & 8 & 8 & 8 & 2 \\
\hline 3-Im & 10000 & STC156 & 575 & 29 & 32 & 16 & 11 & 8 & 44 \\
\hline 4-Im & 10001 & non & 577 & 4 & 3 & 16 & 11 & 8 & 6 \\
\hline 5-Im & 2008 & non & 6 & 6 & 5 & 136 & 11 & 8 & 6 \\
\hline 12-Im & 2008 & non & 6 & 6 & 5 & 136 & 11 & 8 & 6 \\
\hline 14-Im & 10002 & non & 6 & 4 & 503 & 159 & 9 & 23 & 7 \\
\hline 17-Im & 361 & non & 10 & 99 & 5 & 91 & 8 & 7 & 2 \\
\hline 18-Im & 361 & non & 10 & 99 & 5 & 91 & 8 & 7 & 2 \\
\hline 19-Im & 1121 & non & 6 & 4 & 4 & 159 & 9 & 23 & 7 \\
\hline 20-Im & 5442 & non & 468 & 11 & 4 & 8 & 8 & 8 & 2 \\
\hline 21-Im & 3476 & non & 10 & 11 & 4 & 8 & 291 & 8 & 2 \\
\hline 22-Im & 410 & STC23 & 6 & 4 & 12 & 1 & 20 & 18 & 7 \\
\hline 23-Im & 1121 & non & 6 & 4 & 4 & 159 & 9 & 23 & 7 \\
\hline $25-\operatorname{Im}$ & 361 & non & 10 & 99 & 5 & 91 & 8 & 7 & 2 \\
\hline 28-Im & 10003 & non & 10 & 820 & 613 & 91 & 8 & 7 & 2 \\
\hline 32-Im & 10004 & non & 6 & 6 & 5 & 136 & 11 & 8 & 2 \\
\hline $1-\operatorname{Im} 2$ & 2521 & non & 6 & 19 & 3 & 135 & 11 & 8 & 6 \\
\hline $2-\operatorname{Im} 2$ & 4085 & non & 10 & 7 & 5 & 8 & 8 & 35 & 2 \\
\hline $3-\operatorname{Im} 2$ & 392 & non & 6 & 6 & 14 & 18 & 7 & 7 & 71 \\
\hline $4-\operatorname{Im} 2$ & 1080 & non & 6 & 4 & 7 & 9 & 7 & 7 & 56 \\
\hline $5-\operatorname{Im} 2$ & 2035 & non & 6 & 8 & 4 & 225 & 9 & 23 & 7 \\
\hline 7-Im2 & 410 & STC23 & 6 & 4 & 12 & 1 & 20 & 18 & 7 \\
\hline $8-\operatorname{Im} 2$ & 410 & STC23 & 6 & 4 & 12 & 1 & 20 & 18 & 7 \\
\hline $9-\operatorname{Im} 2$ & 410 & STC23 & 6 & 4 & 12 & 1 & 20 & 18 & 7 \\
\hline $10-\operatorname{Im} 2$ & 4085 & non & 10 & 7 & 5 & 8 & 8 & 35 & 2 \\
\hline 11-Im2 & 4085 & non & 10 & 7 & 5 & 8 & 8 & 35 & 2 \\
\hline 12-Im2 & 215 & STC10 & 10 & 11 & 4 & 8 & 8 & 18 & 2 \\
\hline $8-\operatorname{Im} 3$ & 5746 & non & 207 & 744 & 176 & 141 & 170 & 2 & 2 \\
\hline $1-\operatorname{Im} 5$ & 744 & non & 10 & 11 & 135 & 8 & 8 & 8 & 2 \\
\hline $2-\operatorname{Im} 5$ & 4085 & non & 10 & 7 & 5 & 8 & 8 & 35 & 2 \\
\hline $3-\operatorname{Im} 5$ & 58 & STC155 & 6 & 4 & 4 & 16 & 24 & 8 & 14 \\
\hline I-Im6 & 117 & non & 20 & 45 & 41 & 43 & 5 & 32 & 2 \\
\hline $2-\operatorname{Im} 6$ & 117 & non & 20 & 45 & 41 & 43 & 5 & 32 & 2 \\
\hline $4-\mathrm{N}$ & 10005 & STC-23 & 6 & 4 & 12 & 1 & 20 & 12 & 7 \\
\hline $5-\mathrm{N}$ & 10006 & STC-23 & 575 & 4 & 12 & 1 & 20 & 12 & 7 \\
\hline $4-\mathrm{Hb} 2$ & 69 & STC-69 & 21 & 35 & 27 & 6 & 5 & 5 & 4 \\
\hline
\end{tabular}




\begin{tabular}{lccccccccc}
\hline ID & ST & STC & $\boldsymbol{a d k}$ & fumc & $\boldsymbol{g y \boldsymbol { r } \boldsymbol { B }}$ & $\boldsymbol{i c \boldsymbol { d }}$ & $\boldsymbol{m \boldsymbol { d } h}$ & $\boldsymbol{p u r \boldsymbol { A }}$ & $\boldsymbol{r e c \boldsymbol { A }}$ \\
\hline 1-Hb3 & 58 & STC155 & 6 & 4 & 4 & 16 & 24 & 8 & 14 \\
1-L & 117 & non & 20 & 45 & 41 & 43 & 5 & 32 & 2 \\
8-L2 & 10007 & non & 45 & 45 & 36 & 507 & 8 & 294 & 94 \\
2-Hn1 & 58 & STC155 & 6 & 4 & 4 & 16 & 24 & 8 & 14 \\
6-Hn1 & 10008 & non & 6 & 843 & 57 & 1 & 8 & 8 & 2 \\
7-Hn1 & 540 & non & 6 & 7 & 57 & 1 & 8 & 8 & 2 \\
\hline
\end{tabular}

ST numbers from 10000-08 are arbitrary number for the purpose of analysis.

linked to greatest number of isolates. Other ST such as 744, 215 and 761 in this study were revealed as founder and were also found linked to other already reported isolates (Figure 3B, isolates are encircled). Surprisingly, geoBURST analysis revealed that most of the isolates of ST under study (ST88, ST744, ST215, ST761, ST1121, ST10005 and ST410 etc.) were all founders, while other such as ST2035, ST10002 and ST1080 etc. were demarcated as co-founders.

\section{Sequence compositional analysis}

Sequence compositional analysis was performed in order to determine sequence diversity among the seven alleles used for MLST analysis. For this purpose, average GC contents, number of polymorphic sites, haplotype diversity, sites for synonymous and non-synonymous substitution and mean overall distance were determined and mentioned in Table 5. Selection analysis implemented

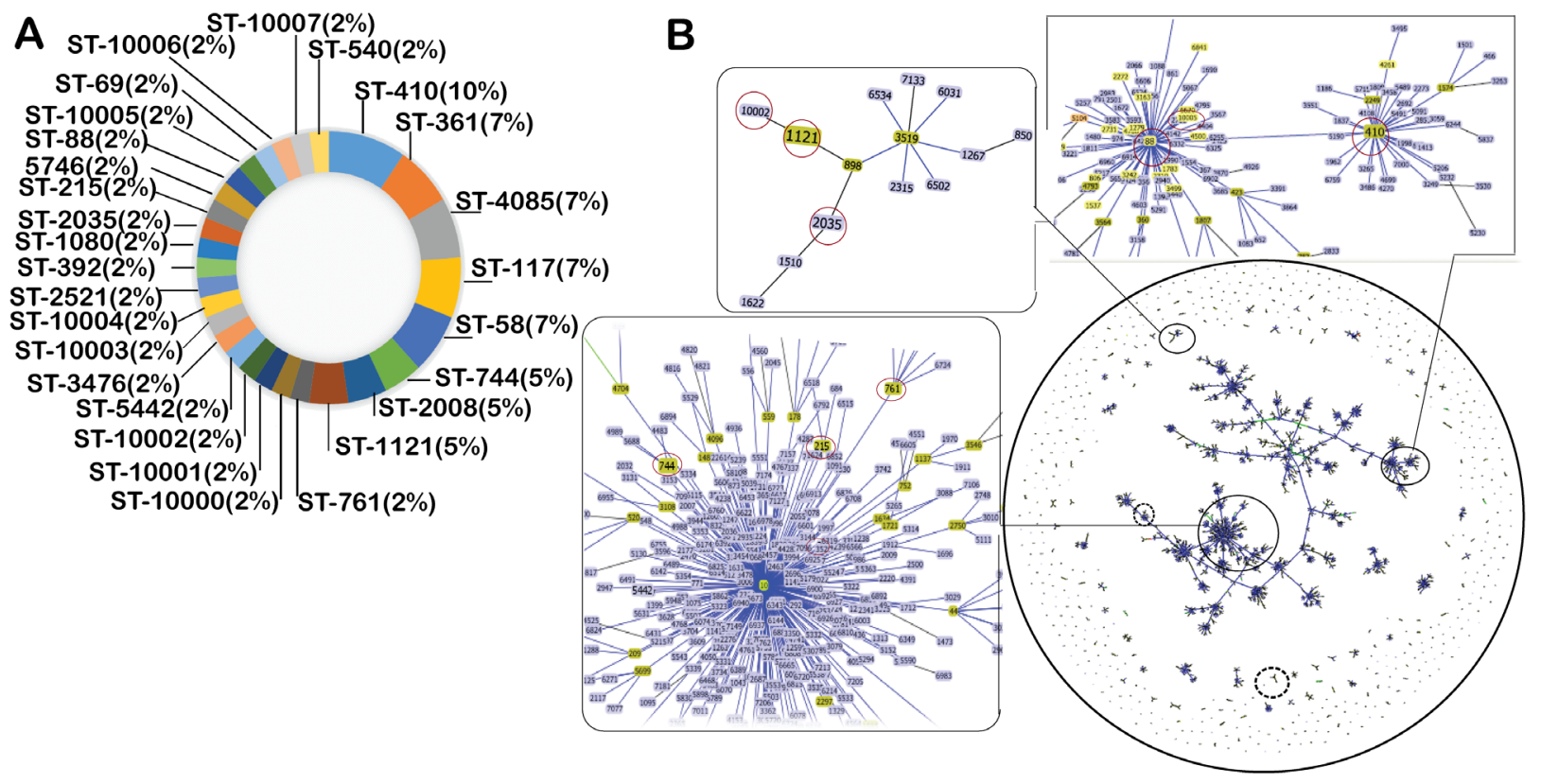

Figure 3: Sequence type diversity and population structure of ESBL-producing isolates. (A) Pie chart representing sequence type diversity and their abundance among the 43 ESBL-positive $E$. coli isolates under study. (B) Population structure of resistant E. coli isolates. The goeBURST analysis of $\sim 7000$ ST available in the PubMLST database against the Achtman scheme. Each dot represents a single ST. Groups of the isolates were formed by linking the ST that are double locus variants (DLV) and named as clonal complex (CC). Snap shots of the three representative CCs zoomed in to indicate our under isolates (encircled). Representative larger size CCs that contain more than three types of our ST are shown with closed circle, while medium size CCs that contain two-to three of ST of our isolates are shown with dashed circle. For clarity reason, groups of CCs that contain a single type of our ST have not been demarcated. Snap shots of the three larger CCs, which are highlighted as closed circle, are zoomed to indicate CCs of our ST that are shown by red-circle. Light green-group founder; dark greensub-group founder; light blue-common node. 
Table 5: Compositional characteristics of genes used in multilocus sequence typing of ESBL-producing $E$. coli

\begin{tabular}{|c|c|c|c|c|c|c|c|c|}
\hline Locus & Length & $\mathbf{G}+\mathbf{C}^{\mathbf{a}}$ & Poly. sites ${ }^{b}$ & $\mathbf{H D}^{\mathbf{c}}$ & $\mathbf{S s}^{\mathrm{d}}$ & $\mathbf{N s}^{\mathrm{e}}$ & $\mathbf{P S}_{\text {prob }}{ }^{\mathrm{f}}$ & d/S.E $E^{g}$ \\
\hline$a d k$ & 536 & 52.9 & 30 & 0.687 & 129.42 & 404.58 & $3.41 / 0.00$ & $1.64 / 2.4$ \\
\hline fumc & 469 & 55.6 & 39 & 0.836 & 109.79 & 355.21 & $5.55 / 0.00$ & $0.01 / 0.01$ \\
\hline$g y r B$ & 460 & 52.8 & 113 & 0.886 & 108.58 & 350.42 & $4.96 / 0.00$ & $0.02 / 0.01$ \\
\hline$i c d$ & 518 & 50.5 & 87 & 0.899 & 127.45 & 388.55 & $5.46 / 0.00$ & $0.02 / 0.01$ \\
\hline$m d h$ & 452 & 51.4 & 13 & 0.828 & 117.32 & 332.68 & $3.17 / 0.00$ & $0.01 / 0.00$ \\
\hline purA & 478 & 52.7 & 9 & 0.818 & 123.70 & 350.30 & $2.71 / 0.00$ & $0.01 / 0.00$ \\
\hline $\operatorname{rec} A$ & 510 & 55.9 & $56 \mathrm{~s}$ & 0.708 & 124.33 & 382.67 & $4.56 / 0.00$ & $0.01 / 0.00$ \\
\hline
\end{tabular}

${ }^{\mathrm{a}}$ Average $\mathrm{G}+\mathrm{C}$ content $(\%)$, ${ }^{\mathrm{b}}$ No of polymorphic sites, ${ }^{\mathrm{c}} \mathrm{HD}=$ Haplotype diversity, ${ }_{\mathrm{d}} \mathrm{Ss}=$ Number of Synonymous sites, ${ }^{\mathrm{e}}$ Number of Non synonymous sites, ${ }^{\mathrm{f} P S_{\text {prob }}}=$ Stat value purifying selection and Probability, ${ }^{\mathrm{g}} \mathrm{d} / \mathrm{S}$.E $=$ distance/standard error (d/S.E).

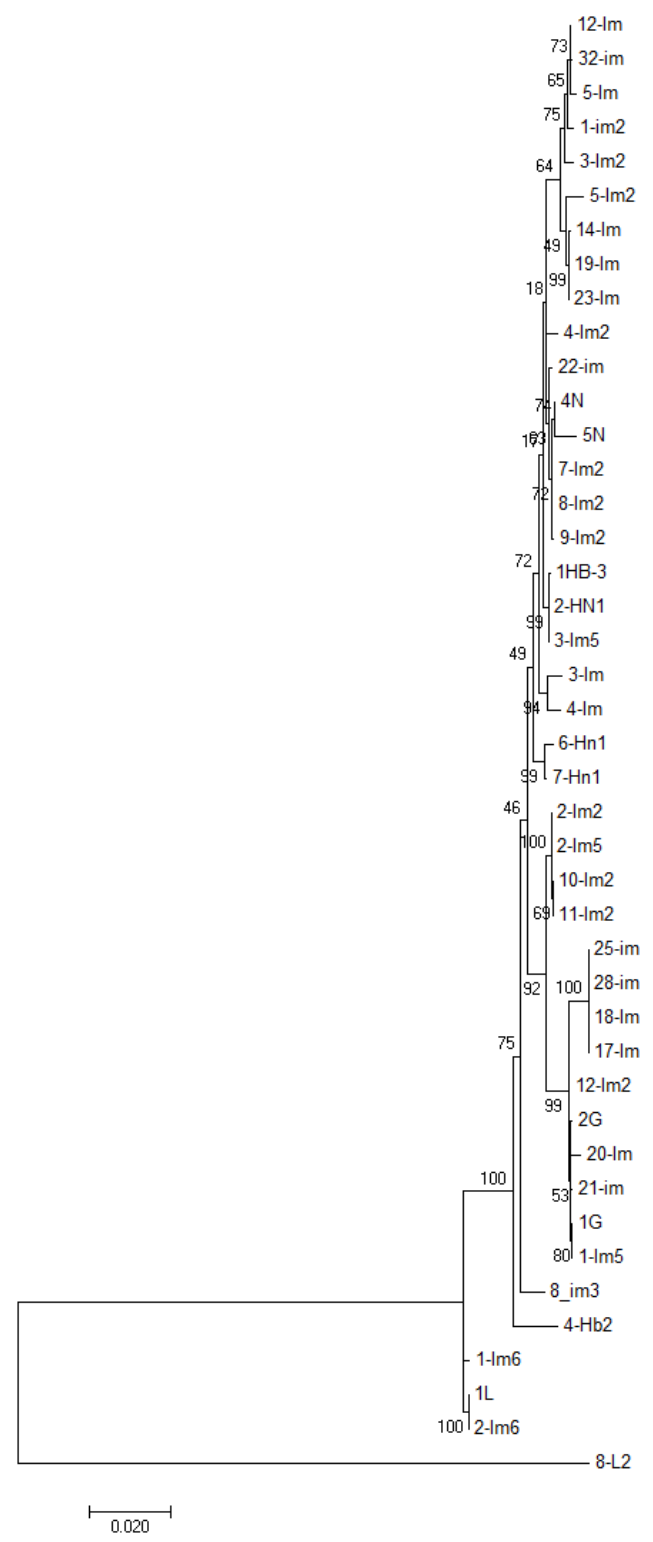

Figure 4: Phylogenetic analysis ESBL-producing $E$. coli isolates $(n=43)$ from bovine mastitis. Phylogenetic tree was constructed from deduced concatenated sequences of the MLST allelic loci with maximum likelihood method using MEGA 7.0. Scale bar is indicated. 
in MEGA 7 programme indicated that all seven loci exhibited purifying selection, therefore, Z-test of selection was performed based on the purifying selection and the stat values are mentioned in Table 5. The mean GC contents of all seven loci were in the range of $50-55.9 \%$, and number of polymorphic sites ranged from 9 to 113 (Table 5).

\section{Multi-locus sequence analysis (MLSA)}

Although we characterized all isolates included in this study into phylogroups, this information does not provide crucial molecular features of the isolates relevant to epidemiological investigation, as well as not allowing accurate identification of species. Thus, to precisely identify species of the $E$. coli isolates under study we implemented MLSA, which is based on analysis of allelic nucleotide sequences used in MLST. Thus, by utilizing the concatenated nucleotide sequences obtained from typed strains $(n=43)$, phylogenetic analysis carried out by using multilocus sequence information of isolates. The maximum likelihood tree constructed using nucleotide sequences of seven loci revealed that study isolates were grouped into two clusters, but the larger cluster had diverse lineage (Figure 4) comprising multiple sub-clusters. A small cluster comprised only of 3 isolates, all originating from Inner Mongolia. The larger cluster was comprised of $42(91 \%)$ isolates, however, unlike the small cluster, this was a tight cluster further comprised of small sub-clusters. An out-group isolate (8-L2) was clustered independently. Interestingly, isolates that could not be assigned with a ST due to a mismatch of at least one of the allelic number; and hence, were assigned with arbitrary numbers (10000-07; 3Im, 4-Im, 28-Im, 32-Im, 4N, 5N, 8L2 and 6-Hn1) (Table 4) were found distributed across all clusters. Furthermore, isolates were not clustered based on the origin or location. Two major sub-clusters, both comprised of a total of 14 isolates each, contained isolates from Guangdong, Hebei, Henan, and Inner Mongolia. However, isolates originating from Jiangsu and Inner Mongolia were clustered on
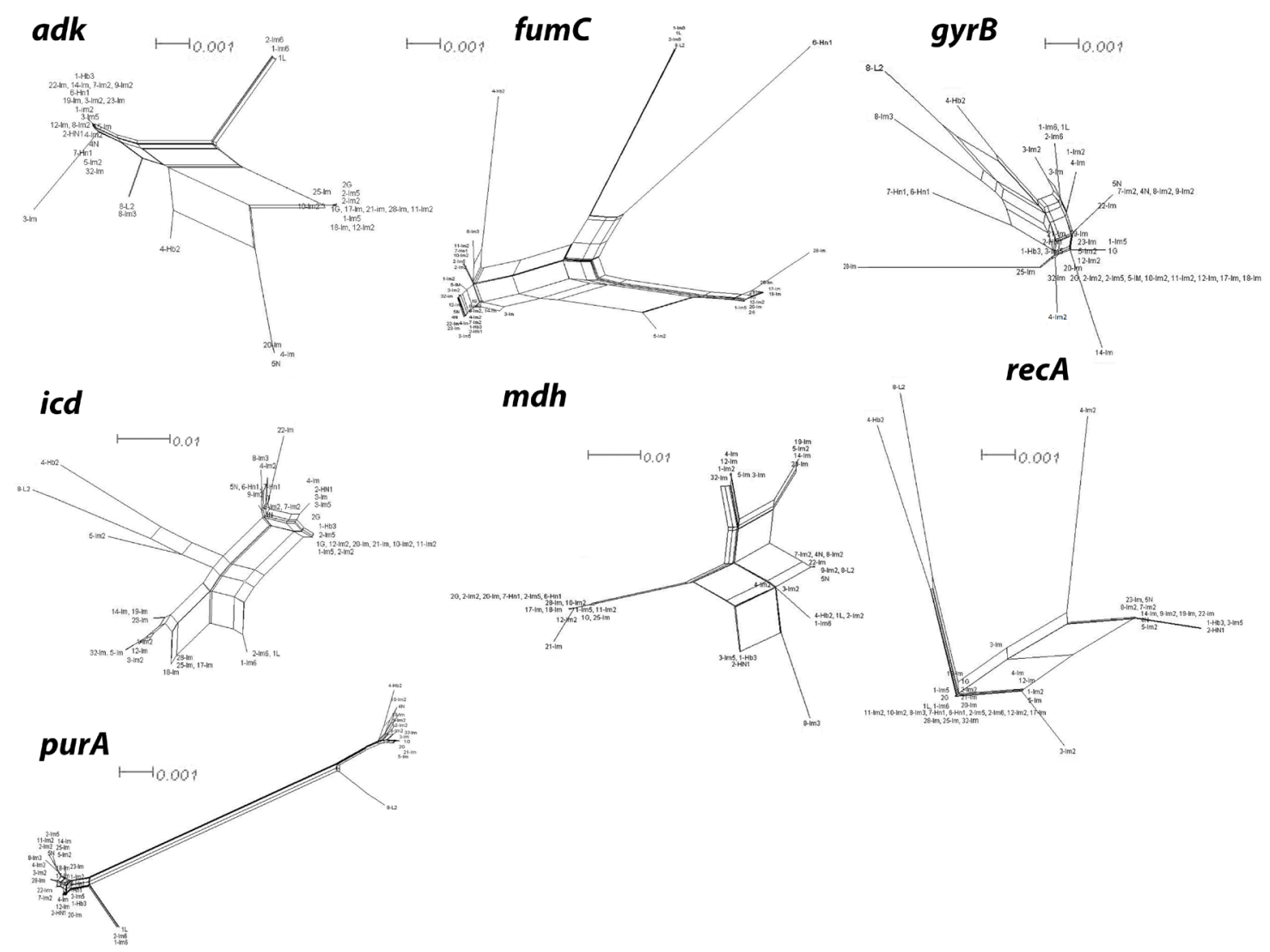

Figure 5: Split network analysis of seven individual multi-locus sequence typing (MLST) loci. Split network analysis of seven individual MLST loci of E. coli isolates under study. Splits Tree4 has been used for creating network graphs by choosing neighbourjoining method. Multi-parallelogram formation with no tree like structures, as seen clearly in the case of adk, fumC, icd, mdh and purA, indicates that recombination has occurred. A tree like network suggests no recombination. Bootstrap values (not shown to avoid clutter) for majority of the parallelograms exceeding $80 \%$ indicating branching is significantly reliable between the strains (branch length of isolate 8-L2 is not drawn to scale). E. coli isolates IDs have been used as numbering in the figure indicate. For ST designations see Table 1. Bar scale is indicated (branch length of isolate 8-L2 is not drawn to scale). 
distinct branches. Isolates from different regions were clustered together, which suggests that a connection between genetic background and sample origin could not be identified (Figure 4).

\section{Recombination analysis}

We performed split network analysis in order to investigate proof of recombination amongst the 43 isolates. Our analysis of $28 \mathrm{ST}$ revealed various structures in the split graphs for the seven loci of MLST (Figure 5). The composition of split graphs of $a d k$, fumc, icd, gyrB and $m d h$ showed network-like parallelogram structures, indicating intergenic recombination happened during the evolutionary history of these genes. The fits were 95.3, 99.4, 97.7, 96.4, 99.2, 96.8 and 99.4 for $a d k$, fumc, gyrB, $i c d, m d h$, purA and recA, respectively, indicating the vast majority of information was consistent with the analysis. All of the parallelograms generated bootstrapping values greater than 50 with majority of them had bootstrapping value greater than 90, which suggests that the analysis were statistically robust. Surprisingly, none of the genes produced an explicit tree-like network which would have revealed these genes were of clonal descent and hence no recombination had occurred. Next, the split decomposition analysis of collective seven MLST loci revealed network-like structures with rays of different lengths (Figure 6). The fit was 78.9 (with LSFit=99.05), indicating that most information was in line with the analysis. The bootstrapping values for parallelogram generated were found greater than $80 \%$ suggesting branching is significantly reliable between the strains. All our tested isolates of $28 \mathrm{ST}$ have been divided into two distinct groups (G1 and G2). Interestingly, both of these groups were completely disconnected from each other suggesting that the interspecies recombination had not happened. Nevertheless, G1, which was the largest group comprised of ST primarily from Inner Mongolia, displayed a parallelogram-shaped network revealing that interspecies intergenic recombination between the isolates of these
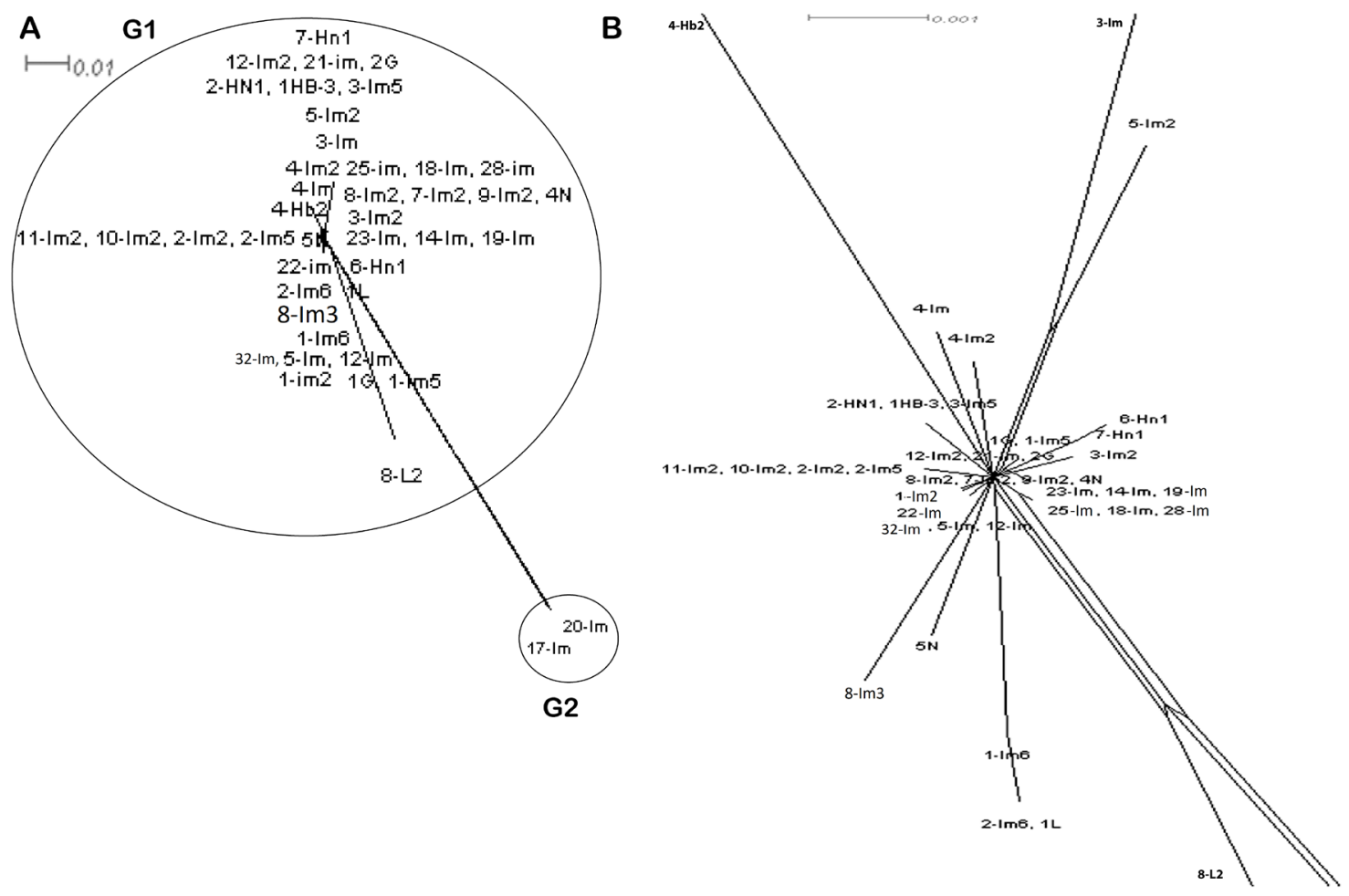

Figure 6: Split network analysis of all seven multi-locus sequence typing allelic loci concatenated sequences of $43 \mathrm{ST}$. Split network analysis based on the concatenated sequence of MLST allelic locus of 43 ST of $E$. coli under study by adopting neighbour-net method implement in Split Tree. (A) Split network analysis of concatenated sequences of MLST allelic locus of $43 \mathrm{ST}$ of $E$. coli isolates indicating a complex network-like structure suggesting several events of recombination. (B) Split network analysis of E. coli lineage belonging to Group 1 and primarily isolates of Inner Mongolia region of China province and reflecting multiparallelogram formation as well complex network-like indicating evidence of recombination. Some of the isolates like 5N, 2-Im6, 1L, 8-Im3 ad 5-Im-2 branched out of the main population network. Bootstrap values (not shown) for all branches are more than $80 \%$. Bar scale is indicated above the figure (branch lengths of 8-L2, 3-Im and 4-Hb2 are not drawn to scale). 
groups had occurred during the course of evolution. ST3951 (isolate 8-L2) is distinct and disconnected from both groups, suggesting that recombination had not occurred between this isolate and the isolates of all other groups (Figure 6). Nevertheless, network structure of the G1 isolates further indicated rays of different lengths and a couple of disconnected isolates.

The phi test, a fast and statistically efficient method, is widely utilized for the analysis of recombination events. The resultant $P$ value produced by applying $p h i$ test for all the 43 isolates of $28 \mathrm{ST}$ is 0.0 (Mean: 0.191, Variance: 1.196, Observed: $0.065, P$-value: 0.0$)$ suggesting significant incidence of recombination across the whole population. Standard association index $\left(I_{A}^{S}\right)$ calculates the homologous recombination by estimating the linkage disequilibrium among the seven MLST loci. When a population is at linkage equilibrium, the resultant $I_{A}{ }^{S}$ is expected to be zero. Analysis of our $43 \mathrm{E}$. coli isolates of 28 ST under this study yielded $I_{A}{ }^{S}$ of $0.2776(P=<0.001)$ (Supplementary Table 3 ). The $I_{A}{ }^{S}$ values are significantly different from zero, suggesting linkage disequilibrium among the alleles showing clonal relationship, and recombination was not adequate to break down the linkage disequilibrium.

\section{DISCUSSION}

In this study, we highlight the features of $46 \mathrm{ESBL}$ producers multi-drug resistant $E$. coli isolated from bovine mastitic milk samples from different provinces of China over the last two years (2015-2016). Among them, 39 isolates harboured CTX-M gene, while 22 and 8 isolates harboured TEM and SHV genes, respectively. These genes were associated with plasmid replicon types, primarily IncF. Interestingly, these resistant genes were transferable as suggested by our mating experiment. In addition, MLSA suggest two major lineages with a large lineage consisted of isolates primarily originated from Inner Mongolia. Split networking and genetic recombination analysis suggested intraspecies recombination events resulted into clonal diversity.

Antimicrobial resistance in Gram-negative bacteria is on the rise worldwide, particularly in $E$. coli, which constitute a majority of invasive Gramnegative isolates. More worrisome is the increasing reports of multi-drug resistant pathogenic E. coli from food producing animals that increases concerns for veterinary and public health [18]. Our study indicates an overall $25.41 \%$ of ESBL prevalence in $E$. coli, which is a greater prevalence of ESBL-producing $E$. coli from bovine mastitis as compared to previous reports from other countries [19, 20]. Possibly, the consistent selective pressure created by the long term use of antibacterial therapy during treatment of mastitis encouraged this rise of ESBL resistance. Our finding of higher prevalence $(39 / 46)$ of bla ${ }_{\text {СтХ-М }}$ genotypes are consistent with our previous report [4] and other reports from China and other parts of the world [3, 20]. Notably, the combination of $b l a_{\text {СTX-M }}+b l a_{\mathrm{TEM}}+b l a_{\mathrm{SHV}}$ has been rarely reported. We presume that this combination of resistance genes with other features associated with highly mobile plasmids types, such as IncF, insertion sequence elements or integrons are responsible for conferring MDR features and the rapid dissemination of these resistance determinants. Some of the isolates under study have been previously characterized for integrons and insertion sequence analysis indicated that integrons type 1 was the most prevalent type [4]. However, the ESBL gene was not found associated with integrons, rather it was found linked to ISCR1 [4]. Similarly, all the new ESBL-producing isolates in the current study, as isolated from bovine mastitis, also belonged to class 1 integrons and harbored similar gene cassettes within the variable regions (data not shown). Thus, association of such mobile elements with the antibacterial-bacterial conferring resistant genes together with conjugative plasmids are extremely alarming. This study indicated that the commensal phylogenetic group A represented the major prevalent phylogroup harbouring ESBL genes and having multidrug resistance [21]. This is worrisome, as these commensals may serve as a reservoir for dissemination of resistance elements, and may in the future embrace virulent traits.

MLST is a precise molecular typing technique that has been successfully used to type more than seven thousand $E$. coli isolates available at MLST data base (http://mlst.warwick.ac.uk/mlst/dbs/Ecoli/). MLST enabled us to type and establish clonal relationship of our isolates and multilocus sequence analysis facilitated us to infer phylogenetic relationship amongst the isolates and species of E. coli isolates. MLST analysis of the 43 successfully typeable $E$. coli strains revealed the extent of diversity within $E$. coli population from the world's most populous country, with a high density of human and animal populations, further coupled with the extensive application of antimicrobial therapy in food animals [4]. Our analysis indicated that all 43 isolates are distributed in $28 \mathrm{ST}$, of which 9 could not be assigned as their allelic profile did not match with the available database. Interestingly, all 9 unassigned isolates reflected a single allele mismatch with the database. These isolates might be of novel types, and hence further studies are under way for whole genome sequencing to identify the ST of these isolates. MLSA analysis indicated that all ST were clustered into two lineages, however, both lineages (a smaller tight lineage and a larger diverse lineage) consisted of isolates from diverse origins and various locations. Therefore, we assumed that the current MLST data of $E$. coli isolates was descriptive at the strain level, however might not be used to infer a relationship between the genetic backgrounds and origin of the isolates. Interestingly, few isolates from Inner Mongolia were included in the smaller tight lineage 
which consisted of isolates from almost all other regions we sampled; yet, isolates from Inner Mongolia, Hebei, Liaoning and Henan were also clustered into the bigger diverse group suggesting that these ESBL producers can be more diverse or ancestral. Furthermore, three identical ST, ST410, from same farm of Inner Mongolia isolated at intervals suggesting chronic infection, which is a classic pattern of mastitis.

Notably, ST410 represents $10 \%$ (4/43) of the typeable population reported in this study. All these isolates originated from Inner Mongolia, the province with very dense farming, however, surprisingly, one of the isolates belong to phylogroup D and B1 each, while two isolates belonged to phylogroup A, reflecting diversity. Further, these isolates harbouring IncFIB $(n=4)$, IncFrep $(n=4), \operatorname{IncK} / \mathrm{B}(n=$ $1)$ and IncN $(n=2)$ plasmid replicon types, respectively. Additionally these isolates were carrying diverse types of ESBL genes (CTX-M-55+ TEM-1 $(n=2)$, CTX-M-15+ SHV-1 $(n=1)$, CTX-M-14 $(n=1)$, while they were all resistant to most commonly used $\beta$-lactam and non- $\beta$-lactam drugs (Table 1). Of note, ST410 is distributed worldwide and is reported in Spain to carry CTX-M and virulence genes isolated from meat and clinical samples [22], in Greece harbouring CTX-M-1 and FIIK replicon type [23], in Pakistan and England producing NDM-1 carbapenemase [24] and China [25]. Other ST that were very prevalent included ST361, ST4085, ST117 and ST58. Recently, ST10, ST58, ST69 E. coli producing CTX-15 type of ESBL were also reported in Lebanese cattle [26]. The geoBURST analysis indicated that of the prevalent ST, primarily ST410 and ST361 including the ST88 (less prevalent) were demarcated as founders. These ST have been isolated from different areas, but primarily from Inner Mongolia. ST88 has been reported worldwide, primarily in Europe [27] and Asia including China [28], making it a well-known species. Of note, none of our E. coli strains belonged to ST131, which is the most prevalent ST in human beings. Nevertheless, $E$. coli producing ESBL CTX-M-15 belonging to non-ST131 are also reported in human beings such as ST410, ST38 and ST10 indicating worldwide presence $[29,30]$. This suggests that these globally disseminated clones and isolates from our study are closely related genetically. Finally, analysis of genetic recombination suggests that despite few allelic loci exhibiting intergenic recombination, genotyping diversity generated is clonal in nature and to investigate interspecies recombination events a larger collection of strains is required as with the current low number of isolates no strong evidence of interspecies recombination was observed.

\section{MATERIALS AND METHODS}

\section{Statement of ethics}

Prior approval to carry out the current work was obtained (dated 26/10/2015) from the departmental ethical committee at College of Veterinary Medicine,
China Agricultural University, Beijing (CAU), and it was carried out according to the standard ethical guide lines implemented at CAU. Additionally, milk samples from the mastitic cows were collected according to the standard procedures of National Mastitis Council (NMC) and with proper consultation of the dairy farm's owner or administration.

\section{Milk sample collection}

Milk samples were collected, as per NMC guide lines, from lactating cows (one sample per cow) suffering from bovine mastitis from 69 dairy herds in 16 provinces of China over a period of two years (2015-2016). These samples were taken in sterile bottles $(50 \mathrm{~mL})$ and transported to our mastitis reference laboratory in ice boxes for the identification of causative agents of mastitis and antibiotic susceptibility testing as described previously [4]. Clinical mastitis was diagnosed on the basis of visual abnormality/inflammation of the mammary gland or its milk secretion, and subclinical mastitis was detected by measuring somatic cell count [32]. The details of collected samples are present in the Supplementary Table 1.

\section{Isolation and identification of isolates and ESBL phenotypes}

A $10 \mu \mathrm{L}$ of milk sample was spread by a sterile cotton swab on the tryptose soy agar (TSA; Difco ${ }^{\mathrm{TM}}$, Becton Dickison, Sparks, MD) augmented with defibrinated sheep blood (5\%) and incubated for $24 \mathrm{~h}$ at $37^{\circ} \mathrm{C}$. The Gram negative, as revealed from standard gram staining, were further streaked onto the MacConkey agar (Difco ${ }^{\mathrm{TM}}$ ) and Eosin methylene blue agars $\left(\right.$ Difco $\left.^{\mathrm{TM}}\right)$ and incubated for further 18-24 h. The dark pink colonies on MCA and green metallic sheen colonies on Eosin methylene blue were the presumptive E. coli, which were further confirmed by API-20E kit (bioMérieux, Marcy I'Etoile, France) and PCR assay. Our previous protocol for the isolation and confirmation of ESBL-producing $E$. coli was adopted for the detection of ESBL-phenotypes [31]. Briefly, the ESBL-producing isolates were confirmed by double-disc synergy test [32], after the initial growth of E. coli on MacConkey agar supplemented with cefotaxime $(2 \mathrm{mg} / \mathrm{L})$. All verified isolates were maintained at $-80{ }^{\circ} \mathrm{C}$ in brain heart infusion broth (Sigma-Aldrich) containing $30 \%$ glycerol until further processing.

\section{In vitro antibiotic susceptibility testing}

Antibiotic susceptibility profiles of all the clinical isolates were tested against a panel of eighteen different antibiotics using the Kirby-Bauer disk diffusion method on Muller-Hinton agar (Difco ${ }^{\mathrm{TM}}$ ) as per standard guidelines of CLSI [32]. The antimicrobial agents included ampicillin, amoxicillin/clavulanic 
acid, cephalexin, cefaclor, cefoxatin, cefotaxime, ceftazidime, cefepime, aztreonam, meropenem, nalidixic acid, chloramphenicol, ciprofloxacin, trimethoprim/ sulphamethoxazole, tetracycline, gentamicin, cefotaxime/ clavulanic acid and ceftazidime/clavulanic acid (BD BBL $^{\mathrm{TM}}$, Oxoid $\left.{ }^{\mathrm{TM}}\right)$. Minimum inhibitory concentrations by broth microdilution method of the selected common antibiotics (ampicillin, cefazolin, cefoxatin, cefotaxime, ceftriaxone, ciprofloxacin, norfloxacin, trimethoprim (BBI Life Sciences Corporation, Shanghai, China), amikacin, chloramphenicol, gentamicin, kanamycin and tetracycline (Amresco, OH, USA)) for the ESBLproducing $E$. coli were also determined in accordance with the guidelines of the CLSI [32]. ESBL negative quality control strain was E. coli ATCC25922, whereas, Klebsiella pneumoniae ATCC700603 was used as ESBL positive control strain [32]. Multidrug resistant isolates were those found resistant to at least three or more categories of antimicrobial agents.

\section{Molecular characterization of ESBL-producing isolates}

The genomic and plasmid DNA from the ESBLproducing isolates were extracted by using a commercial kit (QIAamp DNA minikit, Qiagen, Germany). The ESBL encoding genes were detected using polymerase chain reaction (PCR) assay, which included bla ${ }_{\text {СтХ-М' }}$, bla $_{\mathrm{SHV}}$, bla $_{\mathrm{TEM}}[33]$. The primers used in this study are listed in Supplementary Table 2. The reaction mixture (50 $\mu \mathrm{L})$ consisted of $5 \mu \mathrm{L}$ of $10 \mathrm{X}$ TransStart ${ }^{\circledR}$ Top Taq buffer, $4 \mu \mathrm{L}$ of $2.5 \mathrm{mM}$ deoxynucleoside triphosphate (dNTPs; Transgen, Beijing, China), $0.5 \mu \mathrm{L}$ of TransStart ${ }^{\circledR}$ Top Taq DNA polymerase (2U; Transgen, Beijing, China), $2 \mu \mathrm{L}$ of template DNA, $1 \mu \mathrm{L}$ of each primer $(10 \mu \mathrm{M}$; Sunbiotech, Beijing) and $36.5 \mu \mathrm{L}$ of ultra-pure distilled water. The amplified PCR product was purified (TIANquick Midi purification Kit) and bi-directionally sequenced by the primers used for amplification using an automated sequencer (ABI 3730; Applied Biosystems, Foster City, CA, USA). The obtained sequences were submitted to NCBI data base for homology study (http://www.ncbi. nlm.nih.gov/BLAST/). The already confirmed strains [4] and Klebsiella pneumoniae ATCC 700603 were used as positive control strains in PCR assays. Additionally, the phylogenetic groups of all isolates were detected using the triplex PCR scheme of Clermont et al. [31].

\section{Plasmid replicon typing}

PCR-based plasmid replicon typing, using five multiplex and three singleplex PCR assays, was carried out as described previously by Carattoli et al. [34]. Primer sequences, their amplicon sizes and annealing temperatures are mentioned in Supplementary Table 2.

\section{Resistance transfer experiment}

To investigate the transferability of the ESBL encoding genes and to know that these genes were located on plasmids, a conjugation assay was carried out for randomly selected twelve ESBL-producing E. coli (donor strains) using the sodium azide-resistant $E$. coli J53 strain as recipients. Mating assay was performed on MullerHinton agar (Oxoid) plates as described previously [35]. The transconjugates were finally obtained on MullerHinton agar supplemented with cefotaxime (2 mg/L) and sodium azide $(150 \mathrm{mg} / \mathrm{L})$. All transconjugates were screened for antimicrobial susceptibility by disc diffusion method and MIC of cefotaxime and ceftazidime, ESBL phenotypes and genotypes detection and plasmid replicon typing by methods described above.

\section{Multilocus sequence typing of ESBL-producing E. coli}

To determine the evolutionary relationships of the ESBL-producing isolates, MLST of all the isolates was performed by PCR amplification of the seven housekeeping genes ( $a d k$, fum $C, g y r B, i c d, m d h$, purA and $\operatorname{rec} A$ ) following guidelines of the MLST databases (http://mlst.warwick. ac.uk/mlst/dbs/Ecoli/) as proposed by Wirth et al. (2006) [15]. Allelic profile was determined by the specific allelic profiles (combination of alleles) and ST were assigned based on the combination of seven alleles at MLST database. Sequence types were assigned an arbitrary number if their allelic profile did not match with the available database.

\section{Population structure analysis}

To determine, the clonal complexes of ST eBURST algorithm (htpp://ebusrst.mlst.net) was used by applying eBURSTv3, while Burst group/Clonal complexes were defined as the ST sharing six or more common loci. Furthermore, double locus variants and singletons (ST with at least two allelic mismatches with all other ST) were also defined by eBURST analysis [36]. The eBURST algorithm is structured on a model based on bacterial evolution. In such a model, a single ancestor founder ST gives rise to a subset of closely related ST by a series of continuous diversification through the process of evolution. The relationship between the ST generated in this report with the available ST in the global MLST data base (https://pubmlst.org/bigsdb? db=pubmlst_mlst seqdef\&page=query) was assessed by using geoBURST [37]. Sequence alignments of all seven housekeeping genes of concatenated sequences of all tested isolates under study was performed using MUSCL implemented in MEGA 7 and maximum likelihood tree was developed by choosing General Time Reversible model, Gamma distributed and Invariant sites $(\mathrm{G}+1)$ with 1000 bootstrap replication implemented in MEGA version 7.0 [38]. 


\section{Sequence compositional analysis}

The number of polymorphic sites, haplotype diversity, synonymous and non-synonymous sites were calculated by using DnaSP version 5.10 [39]. Average GC contents of each of the seven alleles, mean overall distance and status of purifying selection were calculated using MEGA version 7 after correcting the frame of the open reading frames of housekeeping genes, when required.

\section{Split network and recombination analysis}

The graphs and analysis of split network of all ST as well as individual locus were produced by applying neighbour-net method using SplitsTree4 [40]. The statistical significance for the parameters to perform evolutionary analysis by SplitsTree has been tested using bootstrap resampling at 1000 and level of fits. The pair wise homoplasy index (phi) test [41] integrated in SplitsTree4 [40] for recombination was performed, whereby the $P$ value $<0.05$ concluding recombination event has occurred.

Linkage disequilibrium analysis based on the allelic profile data obtained for MLST was tested by calculating the standardized index of association $\left(I_{A}^{S}\right)$ using LIAN v3.75 [42] at http://guanine.evolbio.mpg.de/cgi-bin/lian/ lian.cgi.pl. The Monte Carlo method was used in order to evaluate the null hypothesis of complete linkage equilibrium $\left(I_{A}^{S}>0\right.$; presence of linkage disequilibrium or clonality) by choosing 10,000 iterations on the allelic profile of under study isolates.

\section{CONCLUSIONS}

Altogether, resistance profiles, analysis of MIC, plasmid replicon typing, MLST and MLSA analysis provided a great insight into the nature of resistance profiles, mode of dissemination and diversity amongst the $E$. coli ESBL-producers isolated from bovine mastitis samples in China. Our isolates, particularly those unassigned ST, are quite interesting and whole genome sequencing of few candidate isolates will certainly help to understand resistance, virulence associated loci and genomic diversity variation by horizontal gene transfer. Moreover, the current study should be expanded to a larger sample size including other food animal species and community hospitals for effective epidemiological and surveillance reports.

\section{Author contributions}

T. A., S. R., and B. H., designed and conceived the experiments. T. A., J. G., M. S., L. Z., D. H. and S. Z. performed the experiment. T. A. and S. R. performed the analysis. T. A. and S. R. wrote the manuscript. B.
H., P. L. R. and U. S. critically reviewed and revised the manuscript. All the authors read and approved the final manuscript.

\section{CONFLICTS OF INTEREST}

The authors declare no conflicts of interest.

\section{FUNDING}

This research was supported by the National Natural Science Foundation of China (No. 3151101034) and (NO. 31572587), Ministry of Education in China major project (No. 313054), Chinese Twelfth "Five-year" National Science and Technology Support Project (No. 2012BAD12B03), and Specialized Research Fund for the Doctoral Program of Higher Education (SRFDP) State Education Ministry (No. 20120008110042).

\section{REFERENCES}

1. Kempf F, Slugocki C, Blum SE, Leitner G, Germon P. Genomic comparative study of bovine mastitis Escherichia coli. PLoS One. 2016; 11: e0147954.

2. Xu G, An W, Wang H, Zhang X. Prevalence and characteristics of extended-spectrum beta-lactamase genes in Escherichia coli isolated from piglets with post-weaning diarrhea in Heilongjiang province, China. Front Microbiol. 2015; 6: 1103.

3. Yu T, He T, Yao H, Zhang JB, Li XN, Zhang RM, Wang GQ. Prevalence of $16 \mathrm{~S}$ rRNA methylase gene rmtb among Escherichia coli isolated from bovine mastitis in Ningxia, China. Foodborne Pathog Dis. 2015; 12: 770-7.

4. Ali T, ur Rahman S, Zhang L, Shahid M, Zhang S, Liu G, Gao J, Han B. ESBL-Producing Escherichia coli from cows suffering mastitis in China contain clinical class 1 integrons with CTX-M linked to ISCR1. Front Microbiol. 2016; 7: 1931.

5. Liu YY, Wang Y, Walsh TR, Yi LX, Zhang R, Spencer J, Doi Y, Tian G, Dong B, Huang X. Emergence of plasmidmediated colistin resistance mechanism MCR-1 in animals and human beings in China: a microbiological and molecular biological study. Lancet Infect Dis. 2016; 16: 161-8.

6. Hawkey PM, Jones AM. The changing epidemiology of resistance. J Antimicrob Chemother. 2009; 64: i3-i10.

7. Ajiboye RM, Solberg OD, Lee BM, Raphael E, DebRoy C, Riley LW. Global spread of mobile antimicrobial drug resistance determinants in human and animal Escherichia coli and Salmonella strains causing community-acquired infections. Clin Infect Dis. 2009; 49: 365-71.

8. D'Andrea MM, Arena F, Pallecchi L, Rossolini GM. CTXM-type beta-lactamases: a successful story of antibiotic resistance. Int J Med Microbiol. 2013; 303: 305-17. 
9. Lahlaoui H, Khalifa AB, Moussa MB. Epidemiology of Enterobacteriaceae producing CTX-M type extended spectrum $\beta$-lactamase (ESBL). Med Mal Infect. 2014; 44: 400-4.

10. Zhao WH, Hu ZQ. Epidemiology and genetics of CTX-M extended-spectrum $\beta$-lactamases in Gram-negative bacteria. Crit Rev Microbiol. 2013; 39: 79-101.

11. García-Fernández A, Chiaretto G, Bertini A, Villa L, Fortini D, Ricci A, Carattoli A. Multilocus sequence typing of IncI1 plasmids carrying extended-spectrum $\beta$-lactamases in Escherichia coli and Salmonella of human and animal origin. J Antimicrob Chemother. 2008; 61: 1229-33.

12. Maslow JN, Mulligan ME, Arbeit RD. Molecular epidemiology: application of contemporary techniques to the typing of microorganisms. Clin Infect Dis. 1993: 153-62.

13. Keys CJ, Dare DJ, Sutton H, Wells G, Lunt M, McKenna T, McDowall M, Shah HN. Compilation of a MALDITOF mass spectral database for the rapid screening and characterisation of bacteria implicated in human infectious diseases. Infect Genet Evol. 2004; 4: 221-42.

14. Nemoy LL, Kotetishvili M, Tigno J, Keefer-Norris A, Harris AD, Perencevich EN, Johnson JA, Torpey D, Sulakvelidze A, Morris JG. Multilocus sequence typing versus pulsedfield gel electrophoresis for characterization of extendedspectrum beta-lactamase-producing Escherichia coli isolates. J Clin Microbiol. 2005; 43: 1776-81.

15. Wirth T, Falush D, Lan R, Colles F, Mensa P, Wieler LH, Karch H, Reeves PR, Maiden MC, Ochman H. Sex and virulence in Escherichia coli: an evolutionary perspective. Mol Microbiol. 2006; 60: 1136-51.

16. Noller AC, McEllistrem MC, Stine OC, Morris JG Jr, Boxrud DJ, Dixon B, Harrison LH. Multilocus sequence typing reveals a lack of diversity among Escherichia coli O157: H7 isolates that are distinct by pulsed-field gel electrophoresis. J Clin Microbiol. 2003; 41: 675-9.

17. Lacher DW, Steinsland H, Blank TE, Donnenberg MS, Whittam TS. Molecular evolution of typical enteropathogenic Escherichia coli: clonal analysis by multilocus sequence typing and virulence gene allelic profiling. J Bacteriol. 2007; 189: 342-50.

18. Seiffert SN, Hilty M, Perreten V, Endimiani A. Extendedspectrum cephalosporin-resistant Gram-negative organisms in livestock: an emerging problem for human health? Drug Resist Updat. 2013; 16: 22-45.

19. Kar D, Bandyopadhyay S, Bhattacharyya D, Samanta I, Mahanti A, Nanda PK, Mondal B, Dandapat P, Das AK, Dutta TK, Bandyopadhyay S, Singh RK. Molecular and phylogenetic characterization of multidrug resistant extended spectrum beta-lactamase producing Escherichia coli isolated from poultry and cattle in Odisha, India. Infect Genet Evol. 2015; 29: 82-90.
20. Geser NS, Hächler H. Occurrence and characteristics of extended-spectrum b-lactamase (ESBL) producing Enterobacteriaceae in food producing animals, minced meat and raw milk. BMC Vet Res. 2015; 8: 1-9.

21. Suojala L, Pohjanvirta T, Simojoki H, Myllyniemi AL, Pitkala A, Pelkonen S, Pyorala S. Phylogeny, virulence factors and antimicrobial susceptibility of Escherichia coli isolated in clinical bovine mastitis. Vet Microbiol. 2011; 147: $383-8$.

22. López-Cerero L, Egea P, Serrano L, Navarro D, Mora A, Blanco J, Doi Y, Paterson DL, Rodríguez-Baño J, Pascual A. Characterisation of clinical and food animal Escherichia coli isolates producing CTX-M-15 extended-spectrum $\beta$-lactamase belonging to ST410 phylogroup A. Int J Antimicrob Agents. 2011; 37: 365-7.

23. Mavroidi A, Miriagou V, Malli E, Stefos A, Dalekos GN, Tzouvelekis LS, Petinaki E. Emergence of Escherichia coli sequence type 410 (ST410) with KPC-2 $\beta$-lactamase. Int J Antimicrob Agents. 2012; 39: 247-50.

24. Mushtaq S, Irfan S, Sarma J, Doumith M, Pike R, Pitout J, Livermore D, Woodford N. Phylogenetic diversity of Escherichia coli strains producing NDM-type carbapenemases. J Antimicrob Chemother. 2011; 66: 2002-5.

25. Liu Y, Feng Y, Wu W, Xie Y, Wang X, Zhang X, Chen X, Zong Z. First report of OXA-181-producing Escherichia coli in China and characterization of the isolate using whole-genome sequencing. Antimicrob Agents Chemother. 2015; 59: 5022-5.

26. Diab M, Hamze M, Madec JY, Haenni M. High prevalence of non-ST131 CTX-M-15-producing Escherichia coli in healthy cattle in Lebanon. Microb Drug Resist. 2017; 23: 261-6.

27. Randall L, Clouting C, Horton R, Coldham N, Wu G, Clifton-Hadley F, Davies R, Teale C. Prevalence of Escherichia coli carrying extended-spectrum $\beta$-lactamases (CTX-M and TEM-52) from broiler chickens and turkeys in Great Britain between 2006 and 2009. J Antimicrob Chemother. 2011; 66: 86-95.

28. Liu C, Qin S, Xu H, Xu L, Zhao D, Liu X, Lang S, Feng X, Liu HM. New Delhi metallo- $\beta$-lactamase 1 (NDM-1), the dominant carbapenemase detected in carbapenem-resistant Enterobacter cloacae from Henan province, China. PLoS One. 2015; 10: e0135044.

29. Falgenhauer L, Imirzalioglu C, Ghosh H, Gwozdzinski K, Schmiedel J, Gentil K, Bauerfeind R, Kämpfer P, Seifert H, Michael GB. Circulation of clonal populations of fluoroquinolone-resistant CTX-M-15-producing Escherichia coli ST410 in humans and animals in Germany. Int J Antimicrob Agents. 2016; 47: 457-65.

30. Madec JY, Haenni M, Nordmann P, Poirel L. Extendedspectrum $\quad \beta$-lactamase/AmpC-and carbapenemaseproducing Enterobacteriaceae in animals: a threat for 
humans? Clin Microbiol Infect. 2017. doi: 10.1016/j. cmi.2017.01.013.

31. Clermont O, Bonacorsi S, Bingen E. Rapid and simple determination of the Escherichia coli phylogenetic group. Appl Environ Microbiol. 2000; 66: 4555-8.

32. CLSI. (2014). Performance standards for antimicrobial susceptibility testing. Clinical and Laboratory Standard Institute. CLSI document, Wayne, PA, pp. M100-S124.

33. Zhao S, White DG, McDermott PF, Friedman S, English L, Ayers S, Meng J, Maurer JJ, Holland R, Walker RD. Identification and expression of cephamycinase bla(CMY) genes in Escherichia coli and Salmonella isolates from food animals and ground meat. Antimicrob Agents Chemother. 2001; 45: 3647-50.

34. Carattoli A, Bertini A, Villa L, Falbo V, Hopkins KL, Threlfall EJ. Identification of plasmids by PCR-based replicon typing. J Microbiol Methods. 2005; 63: 219-28.

35. Freitag C, Michael GB, Kadlec K, Hassel M, Schwarz $\mathrm{S}$. Detection of plasmid-borne extended-spectrum betalactamase (ESBL) genes in Escherichia coli isolates from bovine mastitis. Vet Microbiol. 2017; 200: 151-6.
36. Feil EJ, Li BC, Aanensen DM, Hanage WP, Spratt BG. eBURST: inferring patterns of evolutionary descent among clusters of related bacterial genotypes from multilocus sequence typing data. J Bacteriol. 2004; 186: 1518-30.

37. Francisco AP, Bugalho M, Ramirez M, Carriço JA. Global optimal eBURST analysis of multilocus typing data using a graphic matroid approach. BMC Bioinformatics. 2009; 10: 152 .

38. Tamura K, Stecher G, Peterson D, Filipski A, Kumar S. MEGA6: molecular evolutionary genetics analysis version 6.0. Mol Biol Evol. 2013; 30: 2725-9.

39. Rozas J, Sánchez-DelBarrio JC, Messeguer X, Rozas R. DnaSP, DNA polymorphism analyses by the coalescent and other methods. Bioinformatics. 2003; 19: 2496-7.

40. Huson DH, Bryant D. Application of phylogenetic networks in evolutionary studies. Mol Biol Evol. 2006; 23: 254-67.

41. Bruen TC, Philippe H, Bryant D. A simple and robust statistical test for detecting the presence of recombination. Genetics. 2006; 172: 2665-81.

42. Haubold B, Hudson RR. LIAN 3.0: detecting linkage disequilibrium in multilocus data. Linkage Analysis. Bioinformatics. 2000; 16: 847-8. 